\title{
INTERSECTIONS AND TRANSFORMATIONS OF COMPLEXES AND MANIFOLDS*
}

\author{
BY \\ SOLOMON LEFSCHETZ
}

\section{INTRODUCTION}

In writing this paper my first objective has been to prove certain formulas on fixed points and coincidences of continuous transformations of manifolds. To this proof for orientable manifolds without boundary is devoted most of the second part, the remainder of which is taken up by a study of product complexes in the sense of $\mathrm{E}$. Steinitz, as they are the foundation on which the proof rests. With suitable restrictions the formulas derived are susceptible of extension to a wider range of manifolds, but this will be reserved for a later occasion. It may be stated that our formulas include and completely generalize the early results due to Brouwer and whatever has been obtained since along the same line. $\dagger$ No such generality would have been possible without that powerful instrument, the product complex.

The principle of the method is best explained by means of a very simple example. Let $f(x)$ and $\varphi(x)$ be continuous and uni-valued functions over the interval 0,1 , and let their values on the interval also lie between 0 and 1 . It is required to find the number of solutions of $f(x)=\varphi(x), 0 \leqq x \leqq 1$.

Graphically the problem is solved by plotting the curvilinear arcs

$$
y=f(x), \quad y=\varphi(x), \quad 0 \leqq x \leqq 1,
$$

and taking their intersections. A slight modification of the functions may change the number of solutions, even make them become infinite in number. However, the difference between the numbers of positive and negative crossings of sufficiently close polygonal approximations to the arcs is a fixed number, their Kronecker index. Its determination is then a partial answer to the question, and indeed seemingly the only possible general answer.

* Presented to the Society under somewhat different title at the Chicago Meeting of April 13, 1923, and the Southwestern Section Meeting of December 1, 1923; received by the editors in November, 1924.

† A good bibliography is found in Kérékjarto's recently published volume Topologie, Berlin, $\mathrm{J}$. Springer. For a list of the most recent titles see a paper by J. W. Alexander, these Tra n s a ctions, vol. 25 (1923), p. 173, to which must be added my notes in the Proceedings of the National Academy of Sciences, vol. 9 (1923), p. 90, vol. 11 (1925), pp. 287, 290, summarizing the results of the present paper. 
The two complexes whose product is taken in this case are the unit segments on the $x$ and $y$ axes, their product being the square whose sides they are. Replace the unit segments by two identical manifolds of $n$ dimensions, $M_{n}$ and $M_{n}{ }^{\prime}$, the square by the $M_{2 n}$ image of their pairs of points (product of the two), the arcs by manifolds on $M_{2 n}$ and the exact situation of Part II is obtained.*

In all questions of the above type, the Kronecker index plays then an essential part. In order to put everything on a solid basis, it seemed vital to discuss thoroughly this index. There is in existence an excellent treatment of it by Hadamard, $\dagger$ leaving little to be desired for euclidean spaces, but distinctly insufficient for general manifolds. Then the Kronecker index is only a special topic in the more interesting and far reaching theory of the intersection of complexes on a manifold, $\ddagger$ needed in any case, to some slight extent, for a good treatment of the index itself. To this theory is devoted most of Part I, of which the chief result is as follows : given several complexes on an orientable manifold $M_{n}$, which do not intersect on each other's boundaries nor on that of $M_{n}$, there exists a well defined cycle of $M_{n}$, their intersection. It is well defined in this sense : no matter how the complexes are approximated by means of straight complexes, the cycle intersection of the latter remains homologous to itself. If the approximating complexes intersect in isolated points there is a definite Kronecker index independent of the mode of approximation.

The independence from covering complexes and related modes of defining straightness has presented some of our most serious difficulties. It is a little surprising that the necessity of freeing the Kronecker index from this vitiating circumstance has never been considered in the literature. That the wider problem has not been attacked is natural enough since intersections of general complexes have been studied but very little if at all. $\S$

- This concept appeared first, applied to the special case of algebraic correspondences, in Severi's paper in the Torino Memorie, vol.54 (1904). Needless to say, Severi did not suspect the analysis situs aspect of the problem, hence did not and could not derive the Hurwitz coincidence formulas. See in this connection Enriques and Chisini, Lezioni sulla Teoria Geometrica delle Equazioni, vol. 3, p. 427, also Chisini, I s ti tu to L o m b a rd o Rendic on ti, ser. 2, vol. 7 (1924), p. 481. Their work is anticipated by my first Note, which seems to have escaped their notice.

† Note to Tannery's Introduction d la Theorie des Fonctions. See also the first chapter of my recent Borel Series Monograph, $L^{\prime}$ Analysis Situs et la Geometrie Algebrique, and my paper in the 1921 Transactions which both contain important applications of the index to algebraic geometry, and finally a very interesting paper by Veblen that has just appeared in these Transactions, vol. 25 (1923); results are recalled in Part I, $\$ 7$ of this paper and derived anew in Part II.

$\ddagger$ Considered and actually applied, I believe, for the first time in my Monograph.

8 Some very important results along that line have been obtained of late by $\mathrm{J}$. W. Alexander. See Proceedings of the National Academy of Sciences, vol. 10 (1924), pp. 99, 101, 493. 


\section{Part I. Questions of Intersection}

\section{§1. Preliminaries}

1. In notation and terminology, we shall follow essentially Veblen's Colloquium Lectures on Analysis Situs. We shall assume the reader fairly familiar with this fundamental work and briefly refer to it as "Coll. Lect." The following designations will recur with special frequency in our paper: $S_{n}=$ euclidean $n$-space; $E_{n}=n$-cell $; C_{n}=n$-dimensional complex; $M_{n}=$ manifold of $n$ dimensions ; $\Gamma_{n}$ (also $\gamma_{n}, \delta_{n}$ in Part II $)=n$-cycle. ${ }^{*}$ The various numerical invariants, the signs $\sim, \equiv$, for congruence or homology, and also the definition of orientation are as in Chapter I of my Borel Series Monograph, L'Analysis Situs et la Géométrie Algébrique. In Part II we shall introduce the sign $\approx$ for homologies with division allowed, that is with zero-divisors neglected.

2. Our ordinary complexes shall be restricted to Veblen's regular type. Such a $C_{n}$ is the homeomorph of an $n$-dimensional polyhedron $\Pi_{n}$ whose faces are all simplicial cells (interiors of simplexes) no two intersecting. The cells of $\Pi_{n}$ define those of $C_{n}$. We shall apply the term rectilinear segment, polygonal or polyhedral configuration, etc., to $C_{n}$, as if it were $\Pi_{n}$ itself, meaning thereby the images of the $\Pi_{n}$ configurations. Distances on $C_{n}$ shall also be measured by reference to $\Pi_{n}$, which for the purpose is assumed immersed in some $S_{n}{ }^{\prime}, n^{\prime} \leqq n$. By a subcomplex of $C_{n}$ we shall mean one made up with cells of $C_{n}$.

$C_{n}$ may be subdivided into new complexes, and this can be carried out so that the cells of the new complex be of diameter $<\epsilon$, assigned. Of importance in this connection is the method of regular subdivision (Coll. Lect., p. 89).

3. We shall define manifolds in accordance with a suggestion due to Veblen (Coll. Lect., p. 92). It amounts essentially to demanding of a $C_{n}$ defining an $M_{n}$ that its cells be grouped about any particular one much as if they were all immersed in an $S_{n}$. It will be found worth while to examine the matter at closer range.

Let $E_{k}, k<n$, be any simplicial cell on $C_{n}, E_{k+1}$ another incident with it (i. e. with $E_{k}$ on its boundary). We define a set $\{e\}$ of elements such that (a) to every $E_{k+1}$ corresponds one and only one $e ;(b)$ to $e$ corresponds together with a given $E_{k+1}$ all others having such a cell in common with it; (c) two elements of the set are said to tend towards one another if and

- Ir set of oriented $n$-circuits in Veblen's terminology. 
only if there are corresponding cells whose vertices opposite to $E_{k}$ tend towards one another. This last condition gives a definition of continuity for $\{e\}$.

Now $C_{n}$ is said to define an $M_{n}$ without boundary if for every $E_{k}$, $0 \leqq k \leqq n-1$, the set $\{e\}$ is homeomorphic to the boundary of an $(n-k)$ cell. The $M_{n}$ with boundary is then defined as in Coll. Lect., p. 88.

To verify the manifold condition we proceed much as in Coll. Lect., pp. 88, 90. We shall show in $\S 3$ (No. 14, Lemma II) that there exists a subdivision $C_{n}{ }^{\prime}$ of $C_{n}$ of which $E_{k}=A_{0} A_{1} \cdots A_{k}$ is a cell, the $A$ 's being its vertices. (Incidentally this method, now quite customary, of naming a cell by its vertices will prove very convenient.) Let $E_{h}=A_{0} A_{1} \cdots A_{h}$ be any cell of $C_{n}{ }^{\prime}$ incident with $E_{k}$. Then $A_{k+1} \cdots A_{h}$ is also a cell of $C_{n}{ }^{\prime}$, and the totality of such cells gives rise to a $C_{n-k-1}$ homeomorphic to $\{e\}$. Hence the manifold condition is equivalent to demanding that all these complexes be homeomorphic to cell boundaries. As the complexes for a given $E_{k}$ are all homeomorphic the verification is really independent of the particular $C_{n}{ }^{\prime}$ chosen.

It is as yet unknown whether for a given $M_{n}$ the manifold condition is verified simultaneously for all defining complexes. We shall therefore agree to consider only defining complexes such that the cells of $M_{n}$ for which the condition is verified have for logical sum one and the same point set.

4. The orientation of a simplicial cell is best defined by the order of naming the vertices (Monograph, p. 13). The oriented $C_{n}$ is a complex as previously understood plus an assigned orientation for each $n$-cell. With the orientation of $E_{n}$ there is attached one for its boundary $(n-1)$-cells. $M_{n}$ is called orientable if its defining $C_{n}$ can be so oriented that every nonbounding $E_{n-1}$ receives opposite orientations from its two adjacent $E_{n}$ 's. This property is independent of the particular defining $C_{n}$. For match the complex with a copy of itself so that corresponding boundary points coincide. There will result a set of $n$-circuits, orientable or not at the same time as $C_{n}$ itself. From the known independence for the circuit (Coll. Lect., pp. 100-102) follows that for $M_{n}$.

5. It is frequently convenient to orient $M_{n}$ by means of a special $n$-cell used as indicatrix, thus: on an $E_{n}=A_{0} A_{1} \cdots A_{n}$ of the defining complex we choose another $E_{n}{ }^{\prime}=B_{0} B_{1} \cdots B_{n}$ reducible to the first by an affine transformation of the common $S_{n}$ with coincidence of vertices in the order named, and instead of assigning the order of the $A$ 's, we do it for the $B$ 's.

Let $x_{1}, x_{2}, \cdots, x_{n}$ be cartesian coördinates on $S_{n}$, the origin being $B_{0}$. Then $E_{n}{ }^{\prime}$ is completely defined if we give ourselves the matrix $H^{\prime}$ of the coördinates of the $B$ 's, it being understood that the $i$ th row corresponds 
to $B_{i}$. Let $E_{n}{ }^{\prime \prime}$ be another indicatrix with the same first vertex $B_{0}$, and let $H^{\prime \prime}$ be the corresponding matrix. Then $E_{n}^{\prime \prime}$ is the indicatrix of $+M_{n}$ or $-M_{n}$ according as the signs of the determinants of $H^{\prime}, H^{\prime \prime}$ (certainly $\neq 0)$ are or are not the same.

Remark. Whenever we derive from $C_{n}$ a new complex $C_{n}{ }^{\prime}$, subdivision of the first, we shall agree to orient each $n$-cell of $C_{n}{ }^{\prime}$ so that it constitutes an indicatrix of the $n$-cell of $C_{n}$ that carries it.

6. With Veblen we shall call singular $k$-cell on $C_{n}$ a point set $\bar{E}_{k}$, of $C_{n}$, uniform and continuous image of an ordinary cell $E_{k}$ which we may as well assume simplicial. Any statement concerning $\bar{E}_{k}$, in particular regarding its orientation or boundary cells, is to be interpreted by reference to $E_{k}$.

Let $E_{k}^{1}, \cdots, E_{k}^{p}$ be sensed cells on $C_{n}$, and let $E_{k-1}^{1}, \cdots, E_{k-1}^{q}$ be their bounding $(k-1)$-cells, all cells being possibly (but not necessarily) singular. We shall extend the term " $k$-complex on $C_{n}$ " to cover a symbol

$$
C_{k}=\sum x_{i} E_{k}^{\prime}
$$

where the $x$ 's are arbitrary integers. The points of $C_{k}$ are those of the $E_{k}$ 's whose $x$ coefficient is not zero plus their limit points. To the cells correspond Poincaré congruences

and for $C_{k}$ by definition

$$
E_{k}^{i} \equiv \sum y_{i j} E_{k-1}^{i}
$$

$$
C_{k} \equiv \sum x_{i} y_{i j} E_{k-1}^{j} \text {. }
$$

The $C_{k-1}$ at the right is the boundary of $C_{k}$. If it reduces to zero, $C_{k}$ is a $k$-cycle. The fact that $C_{k-1}$ is a boundary is also expressed by the homology

$$
C_{k-1} \sim 0
$$

$\left(\bmod C_{n}\right)$

Remark. The boundary (sensed) of an $n$-cell is a cycle; the verification is immediate. Hence, by summation the boundary of a $C_{n}$ is also a cycle.

\section{§2. INTERSECTION OF CELLS}

7. Let $E_{h}, E_{k}, E_{n}$ be simplicial cells, the first two on the third. $E_{h}$ and $E_{k}$ may intersect in various ways. Assume that in the $S_{n}$ of $E_{n}$ the spaces $S_{k}$ and $S_{k}$ which carry the other cells are linearly independent, so that their intersection is an $S_{l}, l=h+k-n$. Grant furthermore that $l \geqq 0$, so that $S_{l}$ is an actual space (possibly a point) and also that the cells themselves intersect. This intersection will consist of an element of $S_{l}$, bounded by a convex polyhedron, and therefore constitutes an $l$-cell $E_{l}$. If we assume the linear spaces of the boundaries of $E_{h}$ and $E_{k}$ also as independent as possible 
from those of the cells themselves, no bounding $(l-1)$-cell of $E_{l}$ (necessarily polyhedral like $E_{l}$ itself) will be an (l-1)-cell of the boundary of $E_{h}$ or $E_{k}$, but will be merely on one of their bounding $(h-1)$-cells or $(k-1)$-cells respectively, and this we shall assume for the present. $E_{l}$ with its bounding polyhedron constitutes an $M_{l}$ decomposable into simplexes, for example by the regular subdivision process. To this $M_{l}$ we now propose to assign an orientation corresponding to given orientations of the other cells as follows. Let $E_{n}{ }^{\prime}=A_{0} A_{1} \cdots A_{n}$ be a small simplicial cell such that $E_{l}{ }^{\prime}=A_{0} A_{1} \cdots A_{l}$ lies on $E_{l}, E_{h}{ }^{\prime}=A_{0} A_{1} \cdots A_{h}$ on $E_{h}$, and $E_{k}{ }^{\prime}=A_{0} A_{1} \cdots A_{l} A_{h+1} \cdots A_{n}$ on $E_{k}$. Let $a_{s} E_{s}{ }^{\prime}, a_{s}= \pm 1$, be the indicatrix of $E_{s}(s=h, k, l, n)$. Then $a_{l}$ is to be determined by the relation

$$
a_{h} \cdot a_{k} \cdot a_{l} \cdot a_{n}=+1 \text {. }
$$

The cell so sensed shall be designated by $E_{h} \cdot E_{k}$.

The relation between the a's shows that if one of the cells $E_{h}, E_{k}, E_{n}$ is inverted so is $E_{l}$. Furthermore if $E_{h}$ and $E_{k}$ are permuted the only indicatrix changed is $E_{n}{ }^{\prime}$, whose vertices undergo $(n-h)(h-l)=(n-h)(n-k)$ transpositions. Hence

$$
E_{h} \cdot E_{k}=(-1)^{(n-h) \cdot(n-k)} E_{k} \cdot E_{h}=-\left(-E_{h}\right) \cdot E_{k}=-E_{h} \cdot\left(-E_{k}\right) .
$$

8. We have tacitly assumed throughout that $l>0$. With suitable conventions we may let it take any value whatever. The case $l<0$ may be dismissed at once; we simply write, then,

$$
E_{h} \cdot E_{k}=0 \text {. }
$$

Let now $l=0$. Then there is a unique point of intersection constituting a zero-cell $E_{0}$. It is this point with the value of $a_{0}$ attached which we designate by $E_{h} \cdot E_{n-h}$. The value of $a_{0}$ is called the Kronecker index or simply index of $E_{h}$ and $E_{n-h}$ and denoted by $\left(E_{h} \cdot E_{n-h}\right)$. The above symbolic relation still holds, and we derive from it and our discussion

$$
\left(E_{h} \cdot E_{n-h}\right)=(-1)^{(n+1) h}\left(E_{n-h} \cdot E_{h}\right)=-\left(-E_{h} \cdot E_{n-h}\right)=-\left(E_{h} \cdot-E_{n-h}\right),
$$

which may also be obtained directly by means of the indicatrices.

To make our conventions complete, when the cells do not intersect, we shall write

$$
E_{h} \cdot E_{k}=0, \quad\left(E_{h} \cdot E_{n-h}\right)=0 .
$$

The case $h=0, k=n$, is not exceptional. We then have a point $E_{0}=A$ and an attached unit $a_{0}$ in place of $a_{h}$. The point is on the "intersecting"

\footnotetext{
" In previous papers the same notation was used, sometimes with, sometimes without the "dot.' In this paper it has been essential to use the dot throughout, for in Part II another "product" symbol comes in, whose meaning is wholly different and which will be written as a "cross" product.
} 
cell $\bar{E}_{n}$ (in place of $E_{k}$ ) and $a_{k}$ is replaced by $\bar{a}_{n}$ whose value is +1 if $E_{n}$ is sensed like $E_{n},-1$ otherwise. The index is now

$$
\left(E_{0} \cdot E_{n}\right)=a_{0}=a_{n} \cdot \bar{a}_{n} \cdot \bar{a}_{0} .
$$

Its sign is that of $\bar{a}_{0}$ if $E_{n}$ and $\bar{E}_{n}$ are sensed alike, its opposite otherwise.

9. Our next task is to determine the boundary congruences. We first assume that $E_{h} \cdot E_{k}$ has no boundary $(l-1)$-cell on the boundary of $E_{n}$. This is indeed the general case, but the exception here referred to is of importance later. Let the boundary congruences for $E_{h}$ and $E_{k}$ be

$$
E_{h} \equiv \sum E_{h-1}^{i}, \quad E_{k} \equiv \sum E_{k-1}^{j} .
$$

The boundary of $E_{h} \cdot E_{k}$ is then the sum of the cells $E_{h} \cdot E_{k-1}^{j}, E_{h-1}^{d} \cdot E_{k}$, affected with signs that are to be determined.

Let for example $E_{h}$ actually intersect $E_{k-1}^{j}$ and choose $E_{n}^{\prime}$ with the vertices of

$$
E_{k-1}^{\prime}=A_{0} A_{1} \cdot \cdot A_{l-1} A_{h+1} \cdot \cdot A_{n} \text { on } E_{k-1}^{j} \cdot
$$

As $A_{l}$ must be transposed $l$ times to come to first place, $(-1)^{l} \cdot a_{k} \cdot E_{k-1}^{\prime}$ is an indicatrix of $E_{k-1}^{j}$. Hence, $a_{k-1}$ corresponding to $E_{k-1}^{j}$ as $a_{k}$ to $E_{k}$, we have

Therefore

$$
a_{k-1}=(-1)^{l} a_{k}
$$

$$
a_{l-1}=(-1)^{l} \cdot a_{l}
$$

corresponds to $E_{h} \cdot E_{k-1}$ as $a_{l}$ to $E_{h} \cdot E_{k}$ itself, so that $E_{h} \cdot E_{k-1}$ has for indicatrix $(-1)^{l} \cdot a_{l} \cdot A_{0} A_{1} \cdots A_{l-1}$, which is the precise indicatrix that it should have as a boundary cell of $E_{h} \cdot E_{k}$, since $A_{l}$ must be transposed $l$ times to be brought to first place, and since $a_{l} A_{0} A_{1} \cdots A_{l}$ is the indicatrix of $E_{h} \cdot E_{k}$.

We conclude then that in the boundary congruence for $E_{h} \cdot E_{k}$ we must affect $E_{h} \cdot E_{k-1}$ with the sign + . Similarly $E_{k} \cdot E_{h-1}$ must be affected with the sign + in the congruence for $E_{k} \cdot E_{h}$, hence $(-1)^{(n-h+1)(n-k)} E_{h-1}^{k} \cdot E_{k}$ with the sign + in the congruence for $(-1)^{(n-h)(n-k)} E_{h} \cdot E_{k}$, from which at once

$$
E_{h} \cdot E_{k} \equiv(-1)^{(n-k)} \sum E_{h-1}^{i} \cdot E_{k}+\sum E_{h} \cdot E_{k-1}^{j} .
$$

In the exceptional case at first excluded, $E_{l}$ will have some bounding $E_{l-1}$ 's on some bounding $E_{n-1}$ of $E_{n}$. This will be due to the fact that for example $E_{n-1}^{p}$ and $E_{k-1}^{q}$ will both lie in $E_{n-1}$. It is found by considering now 
intersections in $E_{n-1}$ that $E_{h-1}^{p} \cdot E_{k-1}^{q}$ is positively related to $E_{h} \cdot E_{k}$. The method is the same as above : we merely assume in our indicatrix $A_{l}$ exterior to $E_{n-1}$ and the rest goes through about as before. We shall then have the general congruence

$$
E_{h} \cdot E_{k} \equiv(-1)^{n-k} \sum E_{h-1}^{i} \cdot E_{k}+\sum E_{h} \cdot E_{k-1}^{j}+\sum E_{h-1}^{p} \cdot E_{k-1}^{q} .
$$

We have here a case where it would be distinctly worth while to have a more complicated notation to indicate in which complex intersections are taken. Such instances are comparatively rare and the doubt will always readily be cleared up by reference to the context.

10. Fundamental theorem on Kronecker indices. Just as before, the case where $l=1$, and the cells at the right are points (zero-cells) offers no exception. $E_{h} \cdot E_{k}$ is then a rectilinear one-cell $E_{1}$ and the three sums at the right reduce to two terms corresponding to the initial and terminal points of $E_{1}$, the sensed intersection. To each of these terms corresponds a Kronecker index, computed either as to $E_{n}$ or as to one of its bounding $(n-1)$-cells. I say, and this is our theorem, that in all cases the sum of these two-indices is zero so that they are units of opposite signs.

11. Let then

$$
E_{1}=E_{h} \cdot E_{n-h+1} \equiv(-1)^{h-1} E_{h-1}^{\prime} \cdot E_{n-h+1}+\cdots,
$$

where we have not written the term that we do not wish to discuss. Assume first that the term written represents the initial point $A_{0}$ of $E_{1}$ and take it for vertex of same name of the indicatrix previously considered. Here then $a_{l}=a_{1}=+1$. The situation is as follows:

$$
\begin{aligned}
& A_{0} A_{2} \cdots A_{h} \quad \text { is indicatrix for } \quad-a_{h} \cdot E_{h-1}^{\prime} \text {, } \\
& A_{0} A_{1} A_{h+1} \cdots A_{n} \quad \text { " " " } a_{n-h+1} \cdot E_{n-h+1} \text {, } \\
& A_{0} A_{2} \cdots A_{h} A_{1} A_{h+1} \cdots A_{n} \text { " " } \quad \text { " }(-1)^{k-1} \cdot a_{n} E_{n} \text {. }
\end{aligned}
$$

The Kronecker index for the point $(-1)^{k-1} \cdot E_{n-1}^{\prime} \cdot E_{n-h+1}$ is then the number $\beta$ defined by the condition

$$
(-1)^{h} \cdot a_{h} \cdot a_{n-h+1} \cdot(-1)^{h-1} \cdot a_{n} \cdot \beta=1 .
$$

We have also

$$
a_{h} \cdot a_{n-h+1} \cdot a_{n} \cdot a_{1}=a_{h} \cdot a_{n-\dot{h}+1} \cdot a_{n}=1 .
$$

Hence finally $\beta=-1$. If $A_{0}$ were the terminal point of $E_{1}$, we would have merely $a_{1}=-1$, the rest being the same, hence $\beta=+1$. Thus we see that the Kronecker indices for the end points have the same signs that the points receive in the boundary congruence for $E_{1}$. The other two cases (where $A_{0}$ is $E_{h} \cdot E_{n-h}$ or $E_{h-1} \cdot E_{n-h}$ and on an $E_{n-1}$ ) lead to exactly the same con- 
clusion; the proofs, essentially similar, are omitted here. The sum of the indices is therefore always zero as was to be proved.

12. The extension to the intersection of $s$ cells $E_{i}, E_{h}, \cdots, E_{k}$ on $E_{n}$, goes through with ease. It is denoted by $E_{i} \cdot E_{h} \cdots E_{k}$, a symbol which obeys the associative but in general not the commutative law. A similar remark holds for the index $\left(E_{i} \cdot E_{h} \cdots E_{k}\right)$, which exists only when $i+h+\cdots+k=n(s-1)$. The boundary congruences can be written down at once.

\section{§3. INTERSECTIONS OF POLYHEDRAL COMPLEXES AND THEIR KRONECKER INDICES}

13. The complexes which are to occupy us in the rest of Part I shall all be immersed in a connected, orientable and oriented manifold $M_{n}$ with an assigned defining complex $C_{n}$. We shall assume throughout that intersecting complexes have non-intersecting boundaries and no common points on the boundary $C_{n-1}$ of $M_{n}$. Of several intersecting complexes so restricted let one, say $C_{h}$, have points on $C_{n-1}$. We may subdivide $C_{h}$ into $C_{h}{ }^{\prime}$ with cells so small that those $h$-cells which have points of $C_{n-1}$, or whose boundary has some, carry no points of the intersecting complexes on themselves or on their boundary. Let $\bar{C}_{h}$ be the complex sum of these cells plus their boundaries. As far as the intersection with the other complexes is concerned, $C_{n}^{\prime}$ may be replaced by $C_{h}{ }^{\prime}-\bar{C}_{h}$ which carries no points of $C_{n-1}$. A similar remark applies in case there are points common to some, but not all, the boundaries of the intersecting complexes. Henceforth it shall then be understood once for all that

I. intersecting complexes have no points on the boundary of $M_{n}$;

II. their boundaries do not meet.

Our general plan is as follows. We shall first define the intersection of a still narrower class of polyhedral complexes, and then approximate general complexes by means of these. But before defining our special polyhedral complexes, we must prove two lemmas.

14. Lemma I. Any polyhedral $C_{h}$ is a sum of simplicial cells.

Each $h$-cell of $C_{h}$ is a sum of a finite number of polyhedral regions of a certain $S_{h}$. Each region is decomposable into a sum of convex polyhedral $h$-cells. ${ }^{*}$ Remove these from $C_{h}$ and let $C_{h-1}$ be the remaining complex. The lemma is true for $h=1$. Grant it for the dimensionality $h-1 ; C_{h-1}$ can then be decomposed into a sum of simplicial cells. Select a point on each convex $h$-cell and join it by rectilinear segments to the simplicial

- This has been proved by Veblen and others. For detailed references see Coll. Lect., p. 83. 
cells of $C_{h-1}$ on the boundary of its $h$-cell. There will follow the requisite decomposition of $C_{h}$.

Remark. A region of the initial decomposition of $C_{h}$ may lie on several cells of $C_{n}$. If so the boundaries of the latter decompose it into regions each of which lies on a unique cell. Then $C_{h}$ will appear as a sum of simplicial cells, each also on a unique cell of $C_{n}$. Whenever we shall consider in the sequel a polyhedral $C_{h}$, arising in some manner in the course of the discussion, we shall assume that it has been decomposed into a sum of simplicial cells each on a unique cell of $C_{n}$. Strictly speaking, the initial complex is thus replaced by a subdivision and should be designated by a new notation, but it will simplify matters a good deal to avoid this.

LEMmA II. There exists a subdivision $C_{n}{ }^{\prime}$ of $C_{n}$ with $C_{h}$ as a sub-complex.

Decompose $C_{h}$ as just stated into a sum of simplicial cells, any one, say $E_{k}$, on an $E_{n}$ of $C_{n}$ or on its boundary. The $S_{k}$ of $E_{k}$ is the intersection of certain $S_{n-1}$ 's of the $S_{n}$ of $E_{n}$. Extend these $S_{n-1}$ 's as far as possible on the simplex of their $E_{n}$. There will result a decomposition of $C_{n}$ into a new complex $\bar{C}_{n}$ with $C_{h}$ as a subcomplex. Apply now Lemma I to $\bar{C}_{n}$ and $C_{n}{ }^{\prime}$ follows.

15. We now seek to define the intersection of two polyhedral complexes $C_{h}, C_{k}$ and its boundary congruences. Let

$$
\begin{array}{ll}
C_{h}=\sum E_{h}^{i} ; & C_{k}=\sum E_{k}^{j} ; \\
E_{h}^{i} \equiv \sum E_{h-1}^{i p} ; & E_{k}^{i} \equiv \sum E_{k-1}^{i q} .
\end{array}
$$

We impose the following restrictive conditions :

(a) Intersecting $h$ - and $k$-cells are on one and the same $n$-cell of $C_{n}$ and in general position as understood in \$2. Their intersection is then an l-cell, where, as before, $l=h+k-n$.

(b) Let $E_{h-1}^{i p}$ intersect $E_{k-1}^{j q}$ on an $E_{l-1}$. Then both are on an $E_{n-1}$ of $C_{n}$ and $E_{l-1}$ is not on the boundaries of $C_{h}$ and $C_{k}$.

When these two conditions are satisfied, the intersection, to be denoted by $C_{h} \cdot C_{k}$, is a $C_{l}$ defined by the relation

$$
C_{h} \cdot C_{k}=\sum E_{h}^{i} \cdot E_{k}^{j},
$$

it being understood that, whenever $E_{h}^{\mathfrak{d}}$ and $E_{\mathbf{k}}^{\jmath}$ do not intersect, $E_{h}^{\mathfrak{s}} . E_{\mathbf{k}}^{\jmath}=0$.

The symbols $C_{h} \cdot C_{k}$ obeys the distributive law, as follows at once from the definition. Thus :

$$
\left(C_{h}{ }^{\prime}+C_{h}{ }^{\prime \prime}\right) \cdot C_{k}=C_{h}{ }^{\prime} \cdot C_{k}+C_{h}{ }^{\prime \prime} \cdot C_{k},
$$


it being granted of course that both $C_{h}{ }^{\prime}$ and $C_{h}{ }^{\prime \prime}$ satisfy conditions $(a)$ and (b) as to $C_{k}$. Similarly for $C_{k}$ while the effect of permuting the $C^{\prime \prime}$ s or inverting them is as in No. 7 for the cells.

The boundary congruences will present no great difficulty. From (9.1) follows

$$
C_{h} \cdot C_{k} \equiv(-1)^{n-k} \sum E_{h-1}^{i p} \cdot E_{k}^{j}+\sum E_{h}^{i} \cdot E_{k-1}^{j q}+\sum E_{h-1}^{i r} \cdot E_{k-1}^{j \bullet},
$$

the meaning of each sum being readily apprehended by reference to (9.1). I say that the terms in the third sum cancel each other. Indeed let $E_{n}^{i}$ and $E_{k}^{j}$ be on the cell $E_{n}$ and give rise to the term $E_{h-1}^{i r} \cdot E_{k-1}^{j s}$, intersection of $E_{h-1}^{i r}, E_{k-1}^{j s}$, situated in a bounding cell $E_{n-1}$ of $E_{n}$. We assume the cells $E_{h-1}^{i r}, E_{k-1}^{j e}, E_{n-1}$ positively related to $E_{h}^{j}, E_{k}^{j}, E_{n}$, and $E_{h-1}^{i r}, E_{k-1}^{j s}$ is the intersection of the two sensed cells, oriented as indicated in $\$ 2$.

According to $(b)$ there exist $E_{n}^{\prime}, E_{n}^{i^{\prime}}, E_{k}^{j^{\prime}}$ of $C_{n}, C_{h}, C_{k}$, with $E_{n-1}, E_{h-1}^{i r}$, $E_{k-1}^{j+}$ on their boundaries and negatively related to them. There is a cell labelled $E_{h-1}^{i r^{\prime}}=-E_{h-1}^{i r}$ and one labelled $E_{k-1}^{j t^{\prime}}=-E_{k-1}^{j s}$. Indeed according to (b) the cells of $C_{h}$ adjacent to $E_{h-1}^{i r}$ can be grouped in pairs oppositely related, and we may assume that we have such a pair in $E_{k}^{i}, E_{h}^{i j}$. Similarly for $E_{k}^{j}$ and $E_{k}^{j^{\prime}}$.

There are now several possibilities. It may be that $E_{n-1}$ separates $E_{k}^{i}$ and $E_{k}^{\prime \prime}$ (that is, one of them is on $E_{n}$, the other on $E_{n}^{\prime}$ ) but not $E_{k}^{j}$ and $E_{k}^{j^{\prime \prime}}$. Then the third sum in (15.1) contains these terms pertaining to the couples considered above and no others :

$$
E_{h-1}^{i r} \cdot E_{k-1}^{j \bullet}+E_{h-1}^{i r} \cdot E_{k-1}^{i \theta^{\prime}} .
$$

They represent intersections on $E_{n-1}$ and as $E_{k-1}^{j \mathbf{s}^{\prime}}=-E_{k-1}^{j s}$ their sum is zero.

A second possibility is that $E_{n-1}$ separates the two pairs of $h$ - and $k$ cells. Then in the sum in question there correspond the terms

$$
E_{h-1}^{i r} \cdot E_{k-1}^{j s}+E_{h-1}^{i^{i r^{\prime}}} \cdot E_{k-1}^{i^{\prime \prime} \theta^{\prime}} \text {. }
$$

The first intersection is taken on $E_{n-1}$, the second on $-E_{n-1}$ (that is in the scheme of $\S 2, E_{n-1}$ must now be replaced by $-E_{n-1}$, the reason being that boundaries of cells on $E_{n}{ }^{\prime}$ are now involved and $E_{n}{ }^{\prime}$ is negatively related to $\left.E_{n-1}\right)$. When intersections are referred to $E_{n-1}$, the second term must be written

$$
-E_{h-1}^{i^{\prime} r^{\prime}} \cdot E_{k-1}^{i^{\prime} 0^{\prime}}=-\left(-E_{h-1}^{i r}\right) \cdot\left(-E_{k-1}^{j \bullet}\right)=-E_{h-1}^{i r} \cdot E_{k-1}^{j \bullet}
$$

and the sum is again zero. 
Finally we must consider the case where $E_{n-1}$ separates none of the two pairs of cells. Then the terms to be considered are now

$$
E_{h-1}^{i r} \cdot E_{k-1}^{i \bullet}+E_{h-1}^{i r} \cdot E_{k-1}^{i_{0}^{\prime}}+E_{h-1}^{i^{i r^{\prime}}} \cdot E_{k-1}^{i \bullet}+E_{h-1}^{i r^{\prime}} \cdot E_{k-1}^{i^{\prime \theta^{\prime}}},
$$

the intersections being all referred to $E_{n-1}$. It is immediately verified that the fourth term is the same as the first, and the other two terms its negative. The sum is then again zero. This completes the proof of our assertion.

16. The boundary of $C_{h}$ is a cycle $\Gamma_{h-1}$, and that of $C_{k}$ is a cycle $\Gamma_{k-1}$. We have

$$
C_{h} \equiv \Gamma_{h-1}, \quad C_{k} \equiv \Gamma_{k-1} .
$$

The first two sums in (15.1) are respectively $\Gamma_{h-1} \cdot C_{k}$ and $C_{h} \cdot \Gamma_{k-1}$. Hence in the last analysis we have this fundamental congruence:

$$
C_{h} \cdot C_{k} \equiv(-1)^{n-k} \Gamma_{h-1} \cdot C_{k}+C_{h} \cdot \Gamma_{k-1} .
$$

17. A series of important corollaries follows at once from the preceding discussion.

I. A complex $C_{h}$ is called a generalized manifold if every non-bounding $E_{h-1}$ of it is incident with just two $h$-cells. Orientability is defined for it as for an ordinary manifold. From our discussion we obtain the following: if $C_{h}$ and $C_{k}$ are orientable generalized manifolds so is $C_{h} \cdot C_{k}$.

II. If one of the complexes is a cycle the boundary of $C_{h} \cdot C_{k}$ is the intersection of this cycle with the other complex or its opposite. If both are cycles so is their intersection. In symbols,

$$
C_{h} \cdot \Gamma_{k}=(-1)^{n-k} \Gamma_{h-1} \cdot \Gamma_{k} ; \quad \Gamma_{h} \cdot C_{k} \equiv \Gamma_{h} \cdot \Gamma_{k-1} ; \quad \Gamma_{h} \cdot \Gamma_{k} \equiv 0 .
$$

III. When the boundary of each complex does not meet the other, $C_{h} \cdot C_{k}$ is a cycle.

IV. Let $\Gamma_{h}$ bound $C_{h+1}$ satisfying our restrictive conditions as to its intersection with $C_{k}$. Then $\Gamma_{h} \cdot C_{k}$ is a bounding cycle also.

This can be read off from (17.1).

18. Kronecker index. This time the dimensions are $h$ and $n-h$. We make the same assumptions as previously, and, in addition, agree that for two non-intersecting cells the index is zero. Then

$$
\left(C_{h} \cdot C_{n-h}\right)=\sum\left(E_{h}^{i} \cdot E_{n-h}^{j}\right) .
$$

From No. 8 follows the distributive law for indices. The result of permuting the two complexes or of changing the sign of one is as for cells and need not be written down. 
From No. 10 and (16.1) it follows that if

$$
C_{h} \equiv \Gamma_{h-1}, \quad C_{n-h+1} \equiv \Gamma_{n-h},
$$

then here

$$
\left(C_{h} \cdot \Gamma_{n-h}\right)=(-1)^{n} \cdot\left(\Gamma_{h-1} \cdot C_{n-h+1}\right),
$$

a formula of great importance later $(\$ 6)$, as it transforms an index corresponding to dimensionalities $h, n-h$, to one with $h$ replaced by $h-1$. $^{*}$

19. From the theorem of No. 10, together with (16.1), now follows

Let $\Gamma_{h}$ bound a $C_{h+1}$ not intersecting the boundary of $C_{n-h}$, a condition that disappears if we deal with a $\Gamma_{n-h}$. Let furthermore the usual restrictions as to intersecting complexes, $C_{n+1}$ and $C_{n-h}$ or $\Gamma_{n-h}$, hold. Then

$$
\left(\Gamma_{h} \cdot C_{n-h}\right)=0,\left(\Gamma_{h} \cdot \Gamma_{n-h}\right)=0 \text {. }
$$

Observe that owing to the distributive law, it is not necessary that $\Gamma_{h} \sim 0$, but merely $\approx 0$, for then $t \Gamma_{h}$ bounds and the multiples of the indices are zero; hence also the indices themselves. This result will have important applications in Part II.

20. The extension to several intersecting complexes offers no particular difficulties. The symbols follow the associative and distributive laws, but not in general the commutative law.

\section{§4. ApPROXImation OF COMPLEXES}

21. A first, but somewhat inelastic, approximation to a general complex $C_{k}$ by a polyhedral $C_{k}{ }^{\prime}$ will be obtained by direct application of processes due to Alexander (these Trans a ction s, vol. 16 (1915), p. 148) and Veblen (Coll. Lect., pp. 95, 118). $C_{k}$ appears then as a subcomplex of a subdivisior of $C_{n}$ with cells of suitably small diameter. There are two associated complexes $C_{k+1}$ and $C_{k}^{0}$ such that

$$
C_{k+1} \equiv C_{k}-C_{k}{ }^{\prime}+C_{k}^{0},
$$

and therefore

$$
C_{k}^{\prime} \sim C_{k}+C_{k}^{0}
$$

$C_{k}^{0}$ appears only when $C_{k}$ is not a cycle. Our $C_{k+1}$ is the same as Veblen's $B_{i+1}$. When $C_{k}$ is not a cycle, the boundary cells of $C_{k+1}$ which join boundary cells of $C_{k}$ and $C_{k}{ }^{\prime}$ are also part of the boundary of $C_{k+1}$, and their sum is precisely $C_{\boldsymbol{k}}^{0}$.

- An analogous formula for cells was given by Veblen in the Transactions paper already quoted. p. 542. 
In the light of this and upon examining the construction, we find by the simplest continuity considerations that it may be carried out so that the following statements hold.

(a) $C_{k}{ }^{\prime}$ and $C_{k+1}$ are both as near as we please to $C_{k}$.

(b) The complex $C_{k}^{0}$ and the boundary of $C_{k}{ }^{\prime}$ are as near as we please to the boundary of $C_{k}$.

(c) $C_{k}{ }^{\prime}$ includes any particular polyhedral boundary subcomplex of $C_{k}$. This is proved by a very transparent application of Lemma II, No. 14 .

22. From the preceding method of approximation we may derive this interesting result: Let the approximation be made by means of cells of $C_{n}$. If the points of a cell of $C_{k}$ are sufficiently near those of $E_{h}$ of $C_{n}$, then its approximation is $E_{h}$ itself or a cell on its boundary. The cell of $C_{k}$ need only be within a certain distance $\delta$ of $E_{h}$ in order that this be true.

From this we have the following

TheOREM. To every polyhedral complex $C_{k}$ corresponds a positive number $\delta$ such that every cycle $\Gamma_{h}$ whose points are all within $\delta$ of $C_{k}$ is homologous to a cycle $\Gamma_{h}{ }^{\prime}$ on $C_{k}$.

For $C_{k}$ is a subcomplex of a subdivision $C_{n}{ }^{\prime}$ of $C_{n}$ (Lemma II, No. 14) and in this case $C_{h}^{0}$ corresponds to $C_{k}^{0}$ of No. 21, for $\Gamma_{h}$ is absent. We can also affirm that $\Gamma_{h}-\Gamma_{h}{ }^{\prime}$ will bound, by $(a)$, a $C_{h+1}$ whose points are as near as we please to $C_{k}$ at the same time as those of $\Gamma_{h}$.

Incidentally, since $C_{k}$ can have no cycle of more than $k$ dimensions, we have this very interesting result: $A$ non bounding cycle cannot be homologous to a cycle as near as we please to a complex of smaller dimensionality.

23. The approximations which we have obtained so far are not flexible enough for our purpose, which demands the approximation of two or more complexes at the same time by others with a well-defined intersection. This will be based upon the all-important

THEOREM. Let $C_{k}$ be a subcomplex of $C_{n}$. By subtraction of bounding $k$-cycles, it may be reduced to another complex with the same boundary, whose $(k-i)$-cells not on the boundary of $M_{n}$ nor on its own are on cells of at least $n-i$ dimensions of $C_{n}$.

Let $C_{n}{ }^{\prime}$ be a regular subdivision of $C_{n}$ and $C_{k}{ }^{\prime}$ the corresponding subdivision of $C_{k}$. Any vertex of $C_{n}{ }^{\prime}$ shall be affected with an upper index, such as $A^{p}$ to indicate the dimensionality $p$ of the cell of $C_{n}$ which carries it.

Let us attach to any cell $E_{r}=A_{0} A_{1} \cdots A_{r}$ of $C_{n}{ }^{\prime}$ a symbol $\left(p_{0}, p_{1}, \cdots, p_{r}\right)$ to describe its type. Observe that the $p^{\prime}$ 's are all distinct, for $p_{0}=p_{1}=p$ would mean that two points on distinct $p$-cells of $C_{n}$ are joined by a rectilinear segment wholly on a $p$-cell. 
Another and more significant property is that the highest $p$ indicates the dimensionality of the cell of $C_{n}$ which carries $E_{r}$. For then $E_{r}$ has points on such a cell (in the vicinity of the corresponding $A^{p}$ ); hence, due to the mechanism of regular subdivision, it lies entirely on it.

Our theorem will then be proved if we can show that $C_{k}$ is reducible to a complex of which every $E_{k-i}$ not on its boundary nor on that of $M_{n}$ has in its symbol an integer $\geqq n-i$.

24. (a) For $n=1$, the reduction is immediate. Then $k=0$ alone needs to be considered. $C_{0}$ is a sum of points each with an assigned sign. Any such point $A$, say affected with + , may be reduced to any point $B$ of $C_{1}$ by adding the end points of a polygonal line $A B$. These end points constitute the bounding $\Gamma_{0}$ of the theorem.

(b) Let now $i=0$ and $E_{k}$ be as yet not reduced. Its symbol is then of type $A_{0}^{p \cdot} \cdots A_{k}^{p k}$ with all the $p^{\text {'s }}$ less than $n$. The cell lies therefore on the boundary of an $n$-cell of $C_{n}$ on which there is a vertex $A_{k+1}^{n}$. Let $\Gamma_{k}$ be the boundary of the simplex $A_{k+1}^{n} A_{0}^{p_{0}} \cdots A_{k}^{p_{k}}$ which is positively related to $E_{k} ; C_{k}{ }^{\prime}-\Gamma_{k}$ is a complex which has the same structure as $C_{k}$ except that $E_{k}$ has been replaced by $k+1$ cells of same dimensionality in every one of whose symbols appears $A_{k+1}^{n}$, so that they are on $n$ cells of $C_{n}$. This carries out the reduction for $i=0$.

(c) Assume that the process goes through for any $M_{n^{\prime}}, n^{\prime}<n$, and also for all cells of more than $k-i=m$ dimensions of $C_{k}{ }^{\prime}$. I say that it goes through for all dimensionalities.

Consider an unreduced $E_{m}=A_{0}^{p_{0}} \cdots A_{m}^{p_{m}}$ of $C_{k}{ }^{\prime}$, the $p^{\prime}$ 's being then all $<n-i=n-k+m$. To any $E_{h}=A_{0}^{p_{0}} \cdots A_{m}^{p_{m}} A_{m+1}^{p_{m+1}} \cdots A_{h}^{p_{h}}$ incident with $E_{m}$ corresponds $E_{h-m+1}=A_{m+1}^{p_{m+1}} \cdots A_{k}^{p h}$ which we sense so that if the first set of $A$ 's is, as we shall assume, an indicatrix of $E_{h}$, the last is one for $E_{h-m-1}$. The totality of these cells is a subcomplex of $C_{k}$ which is an $M_{n-m-1}$ homeomorphic to the boundary of a cell (No.3). The incidence relations (boundary congruences) between corresponding cells are formally identical.

Let $q_{0}, q_{1}, \cdots, q_{n-m-1}$ be the set of integers in increasing order which together with $p_{0}, \cdots, p_{m}$ constitute the set $0,1, \cdots, n$. The manifold $M_{n-m-1}$ carries two defining complexes. The first $C_{n-m-1}$ has the points $A^{q_{0}}$ for vertices, the second $C_{n-m-1}^{\prime}$ the remaining points $A^{q}$. In fact $C_{n-m-1}^{\prime}$ is a regular subdivision of $C_{n-m-1}$, its vertices $q_{i}$ being on $i$-cells of it. To show this it will suffice to examine the relation between those which correspond to $q_{0}$ and $q_{1}$. Let, for example, the sequence of the $p$ 's and $q$ 's in increasing order read $p_{0}, p_{1}, q_{0}, p_{2}, p_{3}, q_{1}, \cdots$, so that $p_{0}=0, p_{1}=1, q_{0}=2$, $\cdots, q_{1}=5, \cdots$. Consider now $E_{2}=A^{0} A^{1} A^{3}$ of $C_{n}{ }^{\prime}$. It is on a certain three-cell of $C_{n}$ on which it is incident with exactly two cells $A^{0} A^{1} A_{1}^{2} A^{3}$ and 
$A^{6} A^{1} A_{2}^{2} A^{3}$ of $C_{n}{ }^{\prime}$. Hence $E_{m+1}=A^{0} A^{1} A^{3} \cdots A^{5} A^{p_{4}} A^{p_{6}} \cdots A^{p_{m}}$ whose symbol is $\left(p_{0}, \cdots, p_{3}, q_{1}, p_{4}, \cdots, p_{m}\right)$ is incident with the two $(m+2)$-cells obtained by placing first $A_{1}^{2}$, then $A_{2}^{2}$ between $A^{1}$ and $A^{3}$. It follows that $A^{3}$ is on the one-cell $A_{1}^{2} A^{3}+A^{3} A_{2}^{2}$ of $C_{n-m-1}$. With the $q$ 's the statement is that $A^{q_{1}}$ is on the one-cell $A_{1}^{q_{0}} A^{q_{1}}+A^{q_{1}} A_{2}^{p_{0}}$. The same reasoning applies to the other $q$ vertices.

Observe that since the $p$ 's are all $<n-k+m$ the $q$ 's must include all integers from $n-k+m$ to $m$. Hence $q_{n-m-1}=n, q_{n-m-2}=n-1, \cdots, q_{n-k-1}=$ $n-k+m$.

25. Let now $\left(p_{0}, \cdots, p_{m} ; q_{0}^{\prime}, \cdots q_{k-m-1}^{\prime}\right)$ be the symbol for any $k$-cell of $C_{k}{ }^{\prime}$ incident with $E_{m}$. The reduction being achieved by assumption for all cells of more than $m$ dimensions, $\left(p_{0}, \cdots, p_{m} ; q_{i}{ }^{\prime}\right)$, the symbol of an $(m+1)$-cell, must possess an integer $\geqq n-k+m+1$ which can only be $q_{i}^{\prime}$. Similarly $\left(p_{0}, \cdots, p_{m} ; q_{i}{ }^{\prime}, q_{j}{ }^{\prime}\right)$ must include at least one integer $\geqq n-k$ $+m+2$, and this can only be $q_{i}^{\prime}$ or $q_{j}^{\prime}$, etc. Finally, then, among the $k-m$ integers $q_{i}^{\prime}$ there must be one at least equal to each integer of the sequence $n-k+m+1, n-k+m+2, \cdots, n$. As they are all distinct and $\leqq n$ they constitute that very sequence and our cell has then the symbol $\left(p_{0}, \cdots\right.$, $\left.p_{m} ; n-k+m+1, \cdots, n\right)$. The symbol of the corresponding cell of $C_{n-m-1}^{\prime}$ is $(n-k+m+1, \cdots, n)$.

26. Let $\bar{C}_{k}$ be the subcomplex of $C_{k}{ }^{\prime}$ which is the sum of its cells incident with $E_{m}$. Since the latter is not on the boundary of $C_{k}{ }^{\prime}$, on any $E_{k-1}$ of $\vec{C}_{k}$, there are as many positively related incident $k$-cells as negatively related. Hence the complex of $C_{n-m-1}^{\prime}$ which corresponds to $\bar{C}_{k}$ is a cycle $\Gamma_{k-m-1}$. Since $C_{n-m-1}^{\prime}$ is homeomorphic to the boundary of a cell, $\Gamma_{k-m-1}$ bounds on the complex, and in fact bounds a subcomplex $C_{k-m}$ of $C_{n-m-1}^{\prime}$ (Coll. Lect., pp. 95, 118). Furthermore since the reduction to be proved applies by assumption to an $M_{n-m-1}, C_{k-m}$ may be so reduced without changing its boundary that in the symbol of its $s$-cells not on the boundary $\Gamma$ there appears $q_{n-k+\ell-1}$ or a higher $q$. But since the cells of $\Gamma$ already satisfy this condition, it holds for all cells of $C_{k-m}$ without exception. From this we conclude immediately that its $(k-m)$-cells have all the same symbol:

$$
\left(q_{n-k-1}, q_{n-k}, \cdots, q_{n-m-1}\right) \equiv(n-k+m, n-k+m+1, \cdots, n) .
$$

That it has this last simple form follows from the remark at the end of No. 24.

27. To $C_{k-m}$ corresponds a subcomplex $C_{k+1}$ of $C_{n}{ }^{\prime}$ whose boundary is a $\Gamma_{k}$. The cells of this cycle incident with $E_{m}$ constitute $\bar{C}_{k}$ so that $E_{m}$ is not a cell of $C_{k}{ }^{\prime \prime}=C_{k}{ }^{\prime}-\Gamma_{k}$. However, among the new cells of $C_{k}{ }^{\prime \prime}$ are found those on $k$-cells of $\Gamma_{k}$ not incident with $E_{m}$ and we must examine these.

The symbol for any $(k+1)$-cell of $C_{k+1}$ is $\left(p_{0}, \cdots, p_{m} ; n-k+m, \cdots, n\right)$. The new $k$-cells introduced have then a symbol such as $\left(p_{0}, \cdots, p_{0-1}\right.$, 
$\left.p_{o+1}, \cdots, p_{m} ; n-k+m, \cdots, n\right)$. In the symbol of any cell of $m+j$ dimensions of its boundary will then appear $n-k+m+j$ or a higher integer. The new cells of $m$ or more dimensions have then the desired behavior. Thus $E_{m}$ and its incident cells have been replaced bv a set of cells which fulfil all requirements. The proof of our theorem is therefore complete.

Remark. We have incidentally obtained the following interesting proposition: Every cycle of a complex defining a manifold without boundary is homologous to a cycle on its dual. More precisely every $\Gamma_{k}$ of $C_{n}$ is homologous to a cycle whose $k$-cells have all the same symbol $(n-k, n-k+1, \cdots, n)$.

28. We return to our approximation problem. The reduction of $C_{k}$ has been obtained by adding the boundaries of $(k+1)$-cells incident with its $k$-cells and belonging to $C_{n}$. Let us replace $C_{n}$ by a subdivision $\bar{C}_{n}$ with cells $<\epsilon$ and $C_{n}{ }^{\prime}$ by a regular subdivision of $\bar{C}_{n}$. If we make the same reduction, we shall merely add to $C_{k}$ the boundaries of $(k+1)$-cells within a distance of $\epsilon$ from the complex.

Applying this reduction to the approximating complex $C_{k}{ }^{\prime}$ we find that we do not thereby disturb (21.1) or (21.2). The complex $C_{k+1}$ is simply increased by cells as near as we please to $C_{k}{ }^{\prime}$, hence to the approximated complex.

29. The essential property of $C_{k}$ is that the $S_{k-i}$ of any $E_{k-i}$ of the complex has the maximum degree of generality relatively to the space of the cell $C_{n}$ that carries $i t$. We mean thereby that, by modifying the complex without changing its cellular structure, $S_{k-i}$ may be brought into coincidence with an arbitrary neighboring $S_{k-\imath}^{\prime}$. The weakest case is when $E_{k-i}$ lies on an $E_{n-i}$ of $C_{n}$ with its vertices on $(n-k)$-cells of the boundary of $E_{n-i}$. Let $A$ be a vertex of $E_{k-i}$ on the cell $E_{n-k} ; S_{k-1}^{\prime}$, will intersect the latter at a point $B$ very near $A$. Impress upon $A$ the rectilinear displacement $A B$, and similarly for the other $k-i$ vertices of $E_{k-i}$, leaving the remaining vertices of $C_{k}{ }^{\prime}$ unchanged. There results an obvious deformation of $C_{k}{ }^{\prime}$, into say $C_{k}{ }^{\prime \prime}$, whereby $S_{k-i}$ is brought into coincidence with $S_{k-1}^{\prime}$ thus proving our assertion.

The displacement of $C_{k}{ }^{\prime}$ may be so carried out, and in a continuous way, that every point will describe a rectilinear path. Their locus is a $\bar{C}_{k+1} \equiv C_{k}{ }^{\prime \prime}-C_{k}{ }^{\prime}$. By adding this to (21.1) we see that $C_{k}{ }^{\prime \prime}$ may take the place of $C_{k}{ }^{\prime}$ with $C_{k+1}+\bar{C}_{k+1}$ in place of $C_{k+1}$. If $S_{k-1}^{\prime}$ is sufficiently near $S_{k-i}$ conditions $(a),(b),(c)$ of No. 21 may still be fulfilled. The reasoning in case $E_{k-i}$ lies on a cell of more than $n-i$ dimensions is the same.

\section{§5. INTERSECTIONS OF GENERAL COMPLEXES}

30. To arrive at something significant, we must narrow down the problem once more. We replace then the second condition of No. 13 by the somewhat more sweeping 
III. Intersecting complexes must not meet the boundaries of one another.

To complexes so restricted we shall ascribe definite cycles or Kronecker indices. Indices and cycles are fixed, in the sense that the former remain the same when we vary the approximating complexes or even the $C_{n}$ by means of which we construct them, while the cycles remain homologous to themselves. We shall at first maintain $C_{n}$ fixed and merely vary the polyhedral approximations, then examine the effect produced by a change of $C_{n}$.

31. Starting first with two complexes $C_{h}, C_{k}$ always restricted as in No. 13, we approximate them as closely as we please by $C_{h}{ }^{\prime}, C_{k}{ }^{\prime}$ constructed as in No. 32 with the system of relations

$$
\begin{array}{lll}
C_{h+1} & \equiv C_{h}-C_{h}+C_{h}^{0} ; & C_{h}^{0} \sim C_{h}+C_{h}^{0} ; \\
C_{k+1} \equiv C_{k}-C_{k}^{\prime}+C_{k}^{0} ; & C_{k}^{\prime} \sim C_{k}+C_{k}^{0} ;
\end{array}
$$

the various complexes have the same meaning as those of similar designation in \$4. If the approximation is sufficiently fine, $C_{h}{ }^{\prime}$ and $C_{k}{ }^{\prime}$ will also fulfil the restrictive conditions I, III of Nos. 13 and 30. This we assume henceforth for all our approximating complexes.

It follows at once from No. 29 that we may so choose $C_{h}{ }^{\prime}$ and $C_{k}{ }^{\prime}$ that they satisfy the two conditions of No. 14 for a well defined intersection $C_{h}{ }^{\prime} \cdot C_{k}{ }^{\prime}$. Since the boundary of each complex does not meet the other, the intersection will be an $l$-cycle. It remains to be shown that this cycle is independent of the approximating complexes.

32. As a preliminary step let $\bar{C}_{h}{ }^{\prime}$ be another approximation whose intersection with $C_{k}{ }^{\prime}$ is well defined. I say that

$$
C_{h}{ }^{\prime} \cdot C_{k^{\prime}} \sim \bar{C}_{h}{ }^{\prime} \cdot C_{k}{ }^{\prime} \text {. }
$$

We have now congruences such as (21.1)

$$
\begin{aligned}
& \bar{C}_{h+1} \equiv C_{h}+\bar{C}_{h}^{0}-\bar{C}_{h}{ }^{\prime}, \\
& C_{h+1} \equiv C_{h}+C_{h}^{0}-C_{h}{ }^{\prime}
\end{aligned}
$$

with $C_{h}^{0}, \bar{C}_{h}^{0}$ very near the boundary of $C_{h}$ and $C_{h+1}, \bar{C}_{h+1}$ very near the complex itself. Their approximation is in fact assumed such throughout that none of these complexes meets the boundary of $C_{k}{ }^{\prime}$. We have then from (32.2) and (32.3)

$$
\bar{C}_{h+1}-C_{h+1} \equiv C_{h}^{\prime}-\bar{C}_{h}^{\prime}-\left(C_{h}^{0}-\bar{C}_{h}^{0}\right) .
$$

To the complex at the left we may apply everything said previously for $C_{\mathrm{k}}$ with the following result. There exists a polyhedral complex $C_{n+1}^{\prime}$ very 
near it, having a well defined intersection with $C_{k}{ }^{\prime}$ and whose boundary is $C_{h}{ }^{\prime}-\bar{C}_{h}{ }^{\prime}$ plus a certain $h$-complex very near $C_{h}^{0}-\bar{C}_{h}^{0}$ and therefore not meeting $C_{k}{ }^{\prime}$. Hence by (16.1)

$$
C_{h+1}^{\prime} \cdot C_{k}^{\prime} \equiv\left(C_{h}^{\prime}-\bar{C}_{h}^{\prime}\right) \cdot \bar{C}_{k}^{\prime} \sim 0 \quad\left(\bmod M_{n}\right),
$$

from which (32.1) follows.

33. Let now $\bar{C}_{h}{ }^{\prime}, \bar{C}_{k}{ }^{\prime}$ be two approximations with a well defined intersection. In order to show that the intersections are independent of the particular polyhedral approximations provided they have a well defined intersection, we must prove that

$$
\dot{C}_{h}{ }^{\prime} \cdot \bar{C}_{k}{ }^{\prime} \sim C_{h}{ }^{\prime} \cdot C_{k^{\prime}} \text {. }
$$

By a slight displacement such as is used in No. 29, we may replace $C_{h}{ }^{\prime}$ by a complex $C_{h}{ }^{\prime \prime}$ with a well defined intersection with both $C_{k}{ }^{\prime}$ and $\bar{C}_{k}{ }^{\prime}$. All that is necessary is to replace throughout $C_{k}{ }^{\prime}$ by $C_{k}{ }^{\prime}-\bar{C}_{k}{ }^{\prime}$. We have then according to the preceding number

$$
C_{h}{ }^{\prime} \cdot C_{k}{ }^{\prime} \sim C_{h}{ }^{\prime \prime} \cdot C_{k}{ }^{\prime} ; \quad \bar{C}_{h}{ }^{\prime} \cdot \bar{C}_{k}{ }^{\prime} \sim C_{h}{ }^{\prime \prime} \cdot \bar{C}_{k}{ }^{\prime} ;
$$

whence (33.1) follows.

$$
C_{h}{ }^{\prime \prime} \cdot C_{k}{ }^{\prime} \sim C_{h}{ }^{\prime \prime} \cdot \bar{C}_{k}{ }^{\prime} \text {, }
$$

Regarding the Kronecker indices, Corollary IV at the end of $\$ 3$ yields at once for $h+k=n$

$$
\left(\left(C_{h}{ }^{\prime}-\bar{C}_{h}{ }^{\prime}-\left(C_{h}^{0}-\bar{C}_{h}^{0}\right)\right) \cdot C_{k}{ }^{\prime}\right)=0,
$$

and as $C_{k}{ }^{\prime}$ does not intersect $C_{h}{ }^{0}-\bar{C}_{h}^{0}$,

$$
\left(C_{h}{ }^{\prime} \cdot C_{k}{ }^{\prime}\right)=\left(\bar{C}_{h}{ }^{\prime} \cdot C_{k}{ }^{\prime}\right)
$$

and the rest is as before.

34. The extension to more than two intersecting complexes is easy. With obvious notations we must show that

$$
C_{h}{ }^{\prime} \cdot C_{k}{ }^{\prime} \cdots C_{l}^{\prime} \sim \bar{C}_{h}{ }^{\prime} \cdot \bar{C}_{k}{ }^{\prime} \cdots \bar{C}_{l}^{\prime} .
$$

Introduce $C_{k}{ }^{\prime \prime}$ in general position as to $C_{k}{ }^{\prime}, \bar{C}_{k}{ }^{\prime}, \ldots, C_{l}{ }^{\prime}, \bar{C}_{l}{ }^{\prime}$. As above it may be shown that in the homology $C_{h}{ }^{\prime}$ and $\bar{C}_{h}{ }^{\prime}$ may both be replaced by $C_{h}{ }^{\prime \prime}$, the process continuing in an obvious way. The treatment for indices is the same.

35. As is natural we denote the cycles and indices defined by means of our approximations as $C_{h} \cdot C_{k} \cdots C_{l}$, or $\left(C_{h} \cdot C_{k} \cdots C_{l}\right)$. These symbols have the same properties as those for polyhedral complexes them- 
selves. Those pertaining to permutation of complexes hold obviously, others less so. We shall examine them in turn, with particular reference to our future needs.

I. Associative law. The scheme of the proof is sufficiently illustrated with three complexes and if we show that

$$
C_{h} \cdot\left(C_{k} \cdot C_{l}\right) \sim C_{h} \cdot C_{k} \cdot C_{l} .
$$

By definition $C_{k} \cdot C_{l}$ and $C_{h} \cdot C_{k} \cdot C_{l}$ are the polyhedral cycles $C_{k}{ }^{\prime} \cdot C_{l}{ }^{\prime}$ and $C_{h}{ }^{\prime} \cdot C_{k}{ }^{\prime} \cdot C_{l}{ }^{\prime}$. In the approximation that leads to the determination of $C_{h} \cdot\left(C_{k}{ }^{\prime} \cdot C_{l}{ }^{\prime}\right)$, the cycle in parentheses can be taken as its own approximation. Hence the cycle at the left in the homology is by definition $C_{h}{ }^{\prime} \cdot\left(C_{k}{ }^{\prime} \cdot C_{l}{ }^{\prime}\right)$ and we are back to the case of polyhedral complexes in general position, for which the law holds.

II. Distributive law. We wish to show that, say,

$$
C_{h} \cdot\left(C_{k}+\bar{C}_{k}\right) \sim C_{h} \cdot C_{k}+C_{h} \cdot \bar{C}_{k} .
$$

On examining the two successive approximations of $\$ 4$ it will be seen that $C_{k}{ }^{\prime}+\bar{C}_{k}{ }^{\prime}$ is an approximation for $C_{k}+\bar{C}_{k}$. If each of the two primed complexes has a well defined intersection with $C_{h}{ }^{\prime}$ so has their sum. Hence the left side is by definition $C_{h}{ }^{\prime} \cdot\left(C_{k}{ }^{\prime}+\bar{C}_{k}{ }^{\prime}\right)$. As the terms at the right are also defined by means of the primed symbols, we are again back to the case of polyhedral complexes where the law holds.

The two preceding proofs hold without modification for the Kronecker index.

III. If $C_{h}, C_{k}, \cdots, C_{l}$ do not actually have a common point, then

$$
C_{h} \cdot C_{k} \cdots C_{l} \sim 0 \text {, or }\left(C_{h} \cdot C_{k} \cdots C_{\imath}\right)=0 \text {. }
$$

For then the primed complexes may be taken without any common point and everything is once more reduced to the known case of polyhedral complexes.

IV. Let $C_{h}$ bound $\bar{C}_{h+1}$ such that it is the only one of the set $\bar{C}_{h+1}, C_{k}, \cdots, C_{l}$ whose boundary may have points in common with the other complexes. Then

$$
C_{h} \cdot C_{k} \cdots C_{l} \sim 0 \text { or }\left(C_{h} \cdot C_{k} \cdots C_{l}\right)=0 \text {. }
$$

From (31.1) follows $\bar{C}_{h+1}-C_{h+1} \equiv C_{h}{ }^{\prime}$, for $C_{h}^{0}$ is now absent since $C_{h}$ is a cycle. Moreover $C_{h}$ being a subcomplex of $\bar{C}_{h+1}$ the sequence $C_{h}, C_{k}, \cdots, C_{t}$ behaves like that of the statement. Let us approximate $\bar{C}_{h+1}-C_{h+1}$ by, say, $C_{n+1}^{\prime}$, in our usual manner, which may be done without changing $C_{h}$ since it is on the boundary (property $(c)$, No. 21). Since $C_{h+1}$ is very near $C_{h}{ }^{\prime}$ 
it will be seen that the primed sequences corresponding to the two considered above behave as they do. The generalization of (16.1) gives here

$$
C_{h+1}^{\prime} \cdot C_{k}{ }^{\prime} \cdots C_{\imath}{ }^{\prime} \equiv C_{h}{ }^{\prime} \cdot C_{k^{\prime}}{ }^{\prime} \ldots . C_{\imath}{ }^{\prime}
$$

from which IV follows.

V. Let $\Gamma_{h} \approx 0$ (i.e., some multiple of $\Gamma_{h} \sim 0$ ). Then

$$
\Gamma_{h} \cdot \Gamma_{k} \cdots \Gamma_{l} \approx 0 \text { or }\left(\Gamma_{h} \cdot \Gamma_{k} \cdots \Gamma_{l}\right)=0 \text {. }
$$

This is an immediate corollary of IV. In both IV and V it is of course not at all necessary that the complex or cycle singled out be the first.

VI. In $C_{h}$ we may suppress any subcomplex not intersecting $C_{k}, \cdots, C_{l}$, without affecting intersection cycle or index.

This is an immediate but important corollary of II and III.

36. Before we proceed with a thorough examination of the effect of passing from $C_{n}$ to a new defining $\bar{C}_{n}$, let us observe that instead of approximating to $C_{h}$ by means of $\bar{C}_{n}$ it is sufficient to do this for $C_{h}{ }^{\prime}$. Indeed, let $\bar{C}_{h}{ }^{\prime}$ be the approximation to $C_{n}{ }^{\prime}$ by means of $\bar{C}_{n}$. We shall have a congruence such as (21.1):

$$
\bar{C}_{h+1} \equiv C_{h}{ }^{\prime}-\bar{C}_{h}{ }^{\prime}+\bar{C}_{h}^{0},
$$

with $\bar{C}_{h+1}$ very near $C_{h}{ }^{\prime}$ and $\bar{C}_{h}^{0}$ very near its boundary. Add this congruence to the first of (31.1) (which is the same as (21.1) with $k$ replaced by $h$ ):

$$
\left(C_{h+1}+\bar{C}_{h+1}\right)=C_{h}-\bar{C}_{h}^{\prime}+\left(C_{h}^{0}+\bar{C}_{h}^{0}\right) .
$$

This is analogous to (21.1) with $\bar{C}_{h}{ }^{\prime}$ as the approximation to $C_{h}$. As the congruences such as (21.1) plus the structure of the approximating complexes themselves were alone used in defining the intersection cycles and indices and deriving their properties, our assertion is proved.

§6. Proof that intersection CyCles and indices are INVARIaNT WHEN THE DEFINING COMPLEX IS CHANGED*

37. For indices the invariance is conditioned upon a certain simple sense convention. Let $C_{n}, \bar{C}_{n}$ be any two defining complexes. By practically the same reasoning as Veblen's in Colloquium Lectures, pp. 101, 102, we may show that one of the two complexes $C_{n} \pm \bar{C}_{n}$ is a bounding cycle, but not both (loc. cit., p. 120). We shall assume in the future that the complexes are so oriented that $C_{n}-\bar{C}_{n}$ is the bounding cycle. Once any particular complex has been assigned an orientation, a definite one follows for the rest.

* A first type of proof is outlined in my second Proceedings note. Just as it appeared in print I discovered the much simpler treatment embodied in this section. 
We must examine if the present convention agrees with our previous mode of sensing a subdivision $C_{n}{ }^{\prime}$ of $C_{n}$, where it will be remembered a cell and its subdivisions were always so sensed as to have a common indicatrix. It is clearly sufficient to consider the case where $C_{n}$ is a simplex with a simplicial subdivision:

$$
C_{n}{ }^{\prime}=\Sigma_{n}^{1}+\cdots+\Sigma_{n}^{p}
$$

Let the first $q$ simplexes, but no others, have an $(n-1)$-simplex on a given simplex $\Sigma_{n-1}$ of $\Sigma_{n}$, namely $\Sigma_{n-1}^{i}$ for $\Sigma_{n}^{i}$, with $A$ and $A^{i}$ as the vertices of $\Sigma_{n}$ and $\Sigma_{n}^{i}$ not on $\Sigma_{n-1}$ or $\Sigma_{n-1}^{i}$. We take the orientations such that $A$ followed by the vertices of $\Sigma_{n-1}$ corresponds to the same as $A_{i}$ followed by those of $\Sigma_{n-1}^{i}$.

In order that $\Sigma_{n}-C_{n}{ }^{\prime}$ be a cycle, it is necessary and sufficient that the boundaries on $\Sigma_{n-1}$ cancel, or that

$$
\Sigma_{n-1}-\left(\Sigma_{n-1}^{1}+\cdots+\Sigma_{n-1}^{q}\right)
$$

be a cycle. This reduces the verification from $n$ to $n-1$, hence ultimately to $n=1$ for which it is immediate.

38. Let us return to two arbitrary defining complexes $C_{n}, \bar{C}_{n}$. Our customary approximations, when applied to $\bar{C}_{n}$ by means of $C_{n}$, cannot go farther than what is yielded by the Alexander-Veblen process. In this case it comes down to this: We subdivide $C_{n}$ and $\bar{C}_{n}$ into $C_{n}{ }^{\prime}$ and $\bar{C}_{n}{ }^{\prime}$, then establish a correspondence $T$ whereby to each cell of $C_{n}{ }^{\prime}$ is assigned a unique one of $C_{n}{ }^{\prime}$. The $n$-cells of $C_{n}{ }^{\prime}$ are each covered positively by $k_{2}$ cells corresponding to positive cells of $\bar{C}_{n}{ }^{\prime}$ and negatively by $k_{1}$ such cells. Furthermore it is a property of the correspondence that $C_{n}{ }^{\prime}-T\left(\bar{C}_{n}{ }^{\prime}\right)$ bounds, hence $k_{2}-k_{1}$ is fixed for all $n$-cells of $C_{n}$, else this complex would have boundary cells exterior to the boundary of $M_{n}$ and would not be a cycle. Moreover

therefore

$$
T\left(\bar{C}_{n}{ }^{\prime}\right) \sim \bar{C}_{n}{ }^{\prime} \sim \bar{C}_{n} \sim C_{n} \sim C_{n}{ }^{\prime} ;
$$

$$
C_{n}{ }^{\prime}-T\left(\bar{C}_{n}{ }^{\prime}\right)=\left(1+k_{1}-k_{2}\right) C_{n}{ }^{\prime} \sim 0 .
$$

When a subcomplex of a $C_{n}$ bounds it bounds also such a subcomplex (Coll. Lect. p. 118). But $C_{n}{ }^{\prime}$ has no $(n+1)$-cells, hence this homology can only be true if $1+k_{1}-k_{2}=0, k_{2}-k_{1}=1$. Thus $T\left(\tilde{C}_{n}{ }^{\prime}\right)$ covers $C_{n}{ }^{\prime}$ exactly once. The importance of this will appear later.

39. Invariance of the index. It will be established by means of (18.1) that

$$
\left(C_{h} \cdot \Gamma_{n-h}\right)=(-1)^{n} \cdot\left(\Gamma_{h-1} \cdot C_{n-h+1}\right),
$$


where the conditions of No. 15 must be satisfied. Here the intersecting complexes are the two extremes, the cycles being their boundaries. We have, then, given $C_{h}, C_{n-h}$, their approximations $C_{h}{ }^{\prime}, C_{n-h}^{\prime}$ by means of a first defining complex $C_{n}$, and $\bar{C}_{h}{ }^{\prime}, \bar{C}_{n-n}^{\prime}$ by a second $\bar{C}_{n}$, the latter being, if we wish, approximations to $C_{h}{ }^{\prime}$ and $C_{n-h}^{\prime}$ rather than to the given complexes (No. 37). We denote the indices as to $C_{n}$ by the usual round parentheses, those as to $\bar{C}_{n}$ by square parentheses, and our object is to show that

$$
\left(C_{h}{ }^{\prime} \cdot C^{\prime}{ }_{n-h}\right)=\left[C_{h}{ }^{\prime} \cdot C_{n-h}^{\prime}\right] \text {. }
$$

Assuming first that neither $h$ nor $n-h$ are zero, we shall replace (39.2) by a similar formula with $h-1$ in place of $h$. This will allow us to reduce everything to the case $h=0$ for which the proof is simple.

40. Suppose that there exists a polyhedral $C_{n-n+1}^{\prime}$ (until further notice straightness and the like are defined by reference to $C_{n}$ ) whose boundary

$$
\Gamma_{n-h}^{\prime}=C_{n-h}^{\prime}+C_{n-h}^{\prime \prime}
$$

where $C^{\prime}{ }_{h}$ and $C_{n-h}^{\prime \prime}$ do not meet. Then first

$$
\left(C_{h}^{\prime} \cdot C_{n-h}^{\prime}\right)=\left(C_{h}^{\prime} \cdot \Gamma_{n-h}^{\prime}\right) .
$$

Also assuming all due conditions satisfied, and denoting by $\Gamma_{h-1}^{\prime}$ the boundary of $C_{h}^{\prime}$, we find from (39.1)

$$
\left(C_{h}^{\prime} \cdot C_{n-h}^{\prime}\right)=(-1)^{h} \cdot\left(\Gamma_{h-1} \cdot C_{n-h+1}^{\prime}\right) .
$$

The necessary conditions are fulfilled with ease. According to our very construction of approximating complexes, $C_{h}^{\prime}$ and $C_{n-h}^{\prime}$ intersect in a finite number of points, any one of which, say $A_{i}$, is on cells of maximum dimensionality of the two complexes and of $C_{n}$. Let in particular $E_{n-h}^{i}$ be that of $C_{n-k}^{\prime}$. We can construct a simplicial $E_{n-h+1}^{i}$ with $E_{n-h}^{i}$ on its boundary and positively related to it. Let this last cell count $m_{i}$ times for $C_{n-n}^{\prime}$. We choose

$$
C_{n-h+1}^{\prime}=\sum m_{i} E_{n-h+1}^{i} ;
$$

$C_{n-h}^{\prime \prime}$ is now what is left of the boundaries of the cells at the right when the cells $m_{i} E_{n-h}^{i}$ are removed from them. We may therefore manifestly so choose the cells $E_{n-h+1}^{i}$ that $C_{n-h}$ will contain no $A$ point.

Without prejudice to what precedes, the cells of $C_{n-h}^{\prime \prime}$ may be brought as near as we please to $C_{n-h}^{\prime}$, hence we may so construct $C_{n-h+1}^{\prime}$ that $C_{n-h}^{\prime \prime}$ intersects $C_{h}$ in a finite number of points (none an $A$ ), each on $h-,(n-h+1)$ and $n$-cells of $C_{h}^{\prime}, C_{n-h+1}^{\prime}$ and $C_{n}$. Let $B$ be such a point. Remove from $C_{h}{ }^{\prime}$ 
a simplicial cell $E_{h}$ containing $B$. If it is sufficiently small, it will contain no $A$ and its bounding $(h-1)$-cells will intersect $C_{n-n+1}^{\prime}$ each at a single point of the cell that carries $B$. Operate similarly for all $B$ 's and let the new complex still be denoted by $C_{h-1}^{\prime}$ and its boundary by $\Gamma_{h-1}^{\prime}$.

If the cells of $C_{n-h+1}^{\prime}$ and those removed from the initial $C_{h}{ }^{\prime}$ are adequately small, the intersection of the new $C_{h}^{\prime}$ with $C_{n-h+1}^{\prime}$ will be a sum of isolated rectilinear segments each on an $n$-cell of $C_{n}$, and as between the two complexes the various restrictions of $\S 3$ are verified. Since the new $C_{h}{ }^{\prime}$ does not meet $C_{n-h}^{\prime \prime}$, it gives rise to the same index $\left(C_{h}^{\prime} \cdot C_{n-h}^{\prime}\right)$ as the initial one, so that we are assured that (40.1) holds with $C_{h}{ }^{\prime}$ as the new approximation to $C_{h}$ and $\Gamma_{h-1}^{\prime}$ as its boundary.

41. At this stage we introduce the second defining complex $\bar{C}_{n}$. All approximations by means of it are to be denoted by the same symbols barred.

We first construct $\bar{\Gamma}_{h-1}^{\prime}, \bar{C}_{n-h}^{\prime}, \bar{C}_{n-h}^{\prime \prime}$. On examining our approximating processes, it is seen that the sum of the last two complexes is a suitable $\bar{\Gamma}_{n-k}^{\prime}$. The cycle $\bar{\Gamma}_{h-1}^{\prime}$ bounds $C_{h}^{\prime}+C_{h}^{0}$, where the second complex, introduced by the approximation, is as near as we please to $\Gamma_{h-1}^{\prime}$. Since $C_{h}^{\prime}$ and $C_{n-h}^{\prime}$ were constructed in general relative position, the second does not meet the boundary $\Gamma_{h-1}^{\prime}$ of the first. With approximations sufficiently fine, it will not meet $C_{h}^{0}$ either. Hence (No. 35, VI)

$$
\left[\left(C_{h}^{\prime}+C_{h}^{0}\right) \cdot C_{n-h}^{\prime}\right]=\left[C_{h}^{\prime} \cdot C_{n-h}^{\prime}\right] .
$$

Therefore in place of approximating $C_{h}{ }^{\prime}$ we may approximate $C_{h}{ }^{\prime}+C_{n}^{0}$. To avoid more notations than necessary, we shall denote that approximation by $\bar{C}_{h}{ }^{\prime}$.

Similarly $\bar{\Gamma}_{n-h}^{\prime}$ bounds a complex $C_{n-h+1}^{\prime}+C_{n-h+1}^{0}$, the latter very near the cycle and therefore not intersecting $\Gamma_{h-1}^{\prime}$, hence, as above, its approximation may take the place of that of $C_{n-h+1}^{\prime}$ and will then be denoted by $\bar{C}_{n-h+1}^{\prime}$.

As previously, we may choose the approximations $\bar{C}_{h}^{\prime}$ and $\bar{C}_{n-h+1}^{\prime}$ such as to fulfil the conditions of $\S 3$ (Nos. 13, 15), for a well defined intersection. Even for $h=1$ is this the case. $\bar{C}_{1}{ }^{\prime}$ may then be so chosen that the points of $\Gamma_{0}{ }^{\prime}$ are approximated by any in their vicinity. We have therefore in all cases where $h>0$

$$
\left[\bar{C}_{h}^{\prime} \cdot \bar{C}_{n-h}^{\prime}\right]=(-1)^{h}\left[\bar{\Gamma}_{h-1}^{\prime} \cdot \bar{C}_{n-h+1}^{\prime}\right] .
$$

By the definition of the index, each expression defines that of the approximated complexes, which are here the primed complexes. Hence

$$
\left[C_{h}^{\prime} \cdot C_{n-h}^{\prime}\right]=(-1)^{h} \cdot\left[\Gamma_{h-1}^{\prime} \cdot C_{n-h+1}^{\prime}\right] .
$$


42. From (40.1) and (41.1) follows that in place of (39.2) we need only prove

$$
\left(\Gamma_{h-1}^{\prime} \cdot C_{n-h+1}\right)=\left[\Gamma_{h-1}^{\prime} \cdot C_{n-h+1}\right],
$$

of the same type but with $h$ replaced by $h-1$. Proceeding thus if necessary we shall reduce $h$ to zero. The last step will consist in replacing $C_{1}{ }^{\prime}$ by its set of terminal points $\Gamma_{0}{ }^{\prime}$, the signs affixed to them being + or - according as they are positively or negatively related to the complex. $\bar{\Gamma}_{0}^{\prime}$ will then consist of points in the same number as those of $\Gamma_{0}^{\prime}$, very near to them and with same signs attached.

For the other complex, we now have a $C_{n}{ }^{\prime}$ and by subdividing $C_{n}$ if necessary, we may assume that $C_{n}{ }^{\prime}$ is a subcomplex of it. Furthermore, (No. 41), the points of $\Gamma_{0}{ }^{\prime}$ may all be chosen on $n$-cells of $C_{n}{ }^{\prime}$.

Owing to the distributive law, we may assume in the last analysis that we have a unique point $A$ with a definite sign affixed, and a unique cell $E_{n}$ of $C_{n}$ carrying the point $A$. We have seen that the sign of the point is to be independent of the approximation; let us assume that it is + . In the other possible case, the reasoning would be the same with perhaps some signs changed. We must then show that

$$
\left(A \cdot E_{n}\right)=+1=\left[A \cdot E_{n}\right] .
$$

Since $A$ is not on $C_{n}-E_{n}$, we may add the last complex to $E_{n}$ without affecting the indices. We have to prove then, that

$$
\left[A \cdot C_{n}\right]=+1 \text {. }
$$

We may choose $A$ on $E_{n}$ not a cell of less than $n$ dimensions of $\bar{C}_{n}$ without affecting our indices. Let $A$ be its own approximation by means of $\bar{C}_{n}$. The approximation of $C_{n}$ by means of $\bar{C}_{n}$ will be the sum of the cells of a certain subdivision of $\bar{C}_{n}$ (No. 40$)$, which is the same as $\bar{C}_{n}$ itself. Finally then

$$
\left[A \cdot C_{n}\right]=\left[A \cdot \bar{C}_{n}\right]=+1,
$$

which completes the proof of the invariance of the index of two complexes.

43. The extension to the index of several complexes can be made along the same lines. The essential thing is to obtain a relation analogous to (40.1). It will be sufficient to derive it for three complexes $C_{h}, C_{k}, C_{l}$, $h+k+l=2 n$, with the usual conditions as to their boundaries. Their approximations will then satisfy conditions analogous to those of No. 15. With obvious notations, let $C_{h+1}^{\prime}$ have for boundary $\Gamma_{h}^{\prime}=C_{h}^{\prime}+C_{h}^{\prime \prime}$ with $C_{h}^{\prime \prime}$ not intersecting $C_{k}{ }^{\prime}$ nor $C_{l}{ }^{\prime}$. As a matter of fact, it would be sufficient to have it not intersecting $C_{k}{ }^{\prime} \cdot C_{l}{ }^{\prime}$. Then

$$
\left(C_{h}{ }^{\prime} \cdot C_{k}{ }^{\prime} \cdot C_{\imath}{ }^{\prime}\right)=\left(\Gamma_{h}{ }^{\prime} \cdot C_{k}{ }^{\prime} \cdot C_{\imath}{ }^{\prime}\right) \text {. }
$$


Also by (16.1) and with $\Gamma_{k-1}^{\prime}$ the boundary of $C_{k}{ }^{\prime}$,

$$
C_{h+1}^{\prime} \cdot C_{k}^{\prime} \equiv(-1)^{n-k} \cdot \Gamma_{h}^{\prime} \cdot C_{k}+C_{h+1}^{\prime} \cdot \Gamma_{k-1}^{\prime},
$$

it being granted that conditions of No. 15 are duly fulfilled. If we now further assume that the boundary of $C_{l}{ }^{\prime}$ does not meet the complex at the left, then (No. 19)

$$
(-1)^{n-k}\left(\Gamma_{h}^{\prime} \cdot C_{k}^{\prime} \cdot C_{l}^{\prime}\right)+\left(C_{h+1}^{\prime} \cdot \Gamma_{k-1}^{\prime} \cdot C_{l}^{\prime}\right)=0
$$

Hence by (43.1)

$$
\left(C_{h}^{\prime} \cdot C_{k}^{\prime} \cdot C_{l}^{\prime}\right)=(-1)^{n-k-1}\left(C_{h+1}^{\prime} \cdot \Gamma_{k-1}^{\prime} \cdot C_{l}^{\prime}\right)
$$

Similarly, with notations whose meaning is transparent,

$$
C_{h}^{\prime} \cdot C_{k}^{\prime} \cdot C_{l}^{\prime}=(-1)^{n-l-1} \cdot\left(C_{h}^{\prime} \cdot C_{k+1}^{\prime} \cdot \Gamma_{l-1}^{\prime}\right) \text {. }
$$

Thus we can raise both $h$ and $k$ at the expense of $l$, until $h=k=n, l=0$. From this point on the details of the discussion differ in no material way from the case of two complexes and need not be given here.

We have then proved that the index of any number of complexes, when any exists, is independent of the defining $C_{n}$ of $M_{n}$ and the related straightness. It has of course the various properties given in No. 36.

44. Invariance of the intersection cycle. The reasoning is exactly the same whatever the number of complexes, so we need only take two, $C_{h}, C_{k}$. This time, with our previous notations, we have to show that

$$
C_{h}^{\prime} \cdot C_{k}^{\prime} \sim \bar{C}_{h}^{\prime} \cdot \bar{C}_{k}^{\prime}
$$

The cycle $C_{h}{ }^{\prime} \cdot C_{k}{ }^{\prime}$ is carried by a $C_{l}$, of the simplest type of No. 2, sum of the distinct simplicial $l$-cells of a decomposition of the cycle into such cells. Let these cells be $E_{l}^{1}, \cdots, E_{l}^{p}$, oriented each in a definite way. We shall have

$$
C_{l}=\sum E_{l}^{i} ; \quad C_{h}^{\prime} \cdot C_{k}^{\prime}=\sum t_{i} E_{l}^{i},
$$

where the $t$ 's are non-zero integers.

Since the barred complexes are as near as we please to the non-barred, $\bar{C}_{h}{ }^{\prime} \cdot \bar{C}_{k}{ }^{\prime}$ is as near as we please to $C_{l}$. Hence (No. 22) the latter carries a $\Gamma_{l}$, subcomplex of a sufficiently fine subdivision, such that $\bar{C}_{h}{ }^{\prime} \cdot \bar{C}_{k}{ }^{\prime}-\Gamma_{l}$ bounds a $C_{l+1}$ whose points are as near as we please to $C_{l}$. But $\Gamma_{l}$ is homo$\operatorname{logous}, \bmod C_{l}$, to a subcomplex of $C_{l}$ itself (Coll. Lect., p. 120). Hence we 
may assume that $\Gamma_{l}$ itself is such a subcomplex without weakening the assertion as to $C_{l+1}$. We shall have

$$
\bar{C}_{h}^{\prime} \cdot \bar{C}_{k}^{\prime} \sim \sum s_{i} E_{l}^{\prime}=\Gamma_{l} .
$$

Owing to our usual approximating procedure, each $E_{l}^{i}$ is on an $n$-cell of $C_{n}$. Hence, just as if $C_{n}$ were an $S_{n}$, we can construct a simplicial $E_{n-\text { i }}$ that intersects $C_{l}$ at a unique point on $E_{l}^{i}$ in such manner that

$$
\left(E_{l}^{i} \cdot E_{n-l}\right)=+1 ; \quad\left(E_{l}^{j} \cdot E_{n-l}\right)=0, \quad j \neq i .
$$

By taking $E_{n-l}$ sufficiently small, we may dispose of it so that its boundary does not meet $C_{l}$, while the cell itself does not meet the boundaries of $C_{h}{ }^{\prime}$ or $C_{k}{ }^{\prime}$. This is due to the fact that the latter do not intersect $C_{l}$ while $E_{n-l}$ is very near to this complex.

Let us now approximate by means of $\bar{C}_{n}$. We have two congruences such as in No. 31 :

$$
\begin{aligned}
& \bar{C}_{h+1} \equiv C_{h}^{\prime}-\bar{C}_{h}^{\prime}+\bar{C}_{h}^{0}, \\
& \bar{C}_{k+1} \equiv C_{k}^{\prime}-\bar{C}_{k}^{\prime}+C_{k}^{0} .
\end{aligned}
$$

When the approximation is carried sufficiently far,

(a) the boundary of $E_{n-l}$ will not meet $C_{l+1}$;

(b) it will not go through any intersection of $\bar{C}_{k+1}$ with $C_{k}{ }^{\prime}$ or $\bar{C}_{k}{ }^{\prime}$, nor of $\bar{C}_{k+1}$ with $C_{k}^{\prime}$ or $\bar{C}_{k}^{\prime}$;

(c) $E_{n-l}$ will not meet $\bar{C}_{h}^{0}$ nor $\bar{C}_{k}^{0}$.

Since $C_{l+1}$ is as near as we please to $C_{l},(a)$ follows from the fact that the boundary does not intersect $C_{l}$. Now were the first of $(b)$ untrue, since $\bar{C}_{h+1}$ is as near as we please to $C_{h}$, it would only mean that the boundary of $E_{n-l}$ goes through a point of $C_{h}{ }^{\prime} \cdot C_{k}{ }^{\prime}$, and hence meets $C_{l}$ which carries this cycle, and this is not the case. Similarly for the rest of $(b)$. As to $(c)$, it follows at once from the fact that the cell does not meet the boundaries of $C_{h}{ }^{\prime}$ and $C_{k}{ }^{\prime}$ to which $\bar{C}_{h}^{0}$ and $\bar{C}_{k}^{0}$ are very near.

Owing to $(a)$ and to IV and I of No. 36, we have

therefore

$$
\left(\left(\Gamma_{l}-\bar{C}_{h}{ }^{\prime} \cdot \bar{C}_{k}{ }^{\prime}\right) \cdot E_{n-l}\right)=0 \text {; }
$$

Also

$$
\sum s_{j}\left(E_{l}^{j} \cdot E_{n-l}\right)=s_{i}=\left(\bar{C}_{h}^{\prime} \cdot \bar{C}_{k}^{\prime} \cdot E_{n-l}\right) .
$$

$$
\left(C_{h}{ }^{\prime} \cdot C_{k}{ }^{\prime} \cdot E_{n-l}\right)=\sum t_{j}\left(E_{l}^{\prime} \cdot E_{n-l}\right)=t_{i} \text {. }
$$

Again by IV and the first of $(b)$,

$$
\left(\left(C_{h}^{\prime}-\bar{C}_{h}^{\prime}+\bar{C}_{h}^{0}\right) \cdot C_{k}^{\prime} \cdot E_{n-l}\right)=0 .
$$


From (c) we have

Therefore

$$
\left(\bar{C}_{h}^{0} \cdot C_{k}{ }^{\prime} \cdot E_{n-l}\right)=0 .
$$

$$
\left(C_{h}{ }^{\prime} \cdot C_{k}{ }^{\prime} \cdot E_{n-l}\right)=\left(\bar{C}_{h}{ }^{\prime} \cdot C_{k}{ }^{\prime} \cdot E_{n-l}\right) .
$$

Similarly from the last of $(b)$ and $(c)$,

Therefore

$$
\left(\bar{C}_{h}{ }^{\prime} \cdot\left(C_{k}{ }^{\prime}-\bar{C}_{k}{ }^{\prime}+\bar{C}_{k}^{0}\right) \cdot E_{n-l}\right)=0 \text {. }
$$

$$
\left(\bar{C}_{h}{ }^{\prime} \cdot C_{k}{ }^{\prime} \cdot E_{n-l}\right)=\left(\bar{C}_{h}{ }^{\prime} \cdot \bar{C}_{k}{ }^{\prime} \cdot E_{n-l}\right) .
$$

By comparing we have at once

$$
t_{i}=\left(C_{h}{ }^{\prime} \cdot C_{k}{ }^{\prime} \cdot E_{n-l}\right)=\left(\bar{C}_{h}{ }^{\prime} \cdot \bar{C}_{k}{ }^{\prime} \cdot E_{n-l}\right)=s_{i} .
$$

From this follows finally

$$
C_{h}{ }^{\prime} \cdot C_{k}{ }^{\prime} \sim \bar{C}_{h}{ }^{\prime} \cdot \bar{C}_{k}{ }^{\prime},
$$

and our invariance proof is complete.

45. Intersection cycles or Kronecker indices are then the same whatever the defining complex of $M_{n}$ from which they are derived, provided as regards indices that various complexes have their orientations suitably related. Cycles and indices have all the properties established in No. 35 and the effect of a permutation of complexes in the symbols is the same as for cells.

\$7. Fundamental SETS ON AN ORIENTABle $M_{n}$ WITHOUT BOUNDARY

46. Let $\Gamma_{h}^{i}\left(i=1,2, \cdots, p_{h}\right), \Gamma_{n-h}^{j}\left(i=1,2, \cdots, p_{n-h}\right)$ be two fundamental sets (No. 7) for the cycles of the same dimensions, and consider the matrix

$$
\left\|\left(\Gamma_{h}^{i} \cdot \Gamma_{n-h}^{j}\right)\right\| \text {. }
$$

Any other fundamental set, say for the dimensionality $h$, can be derived from a given one by applying to its cycles a transformation of determinant unity. This is at once derivable from the result established by Veblen, loc. cit., p. 117. By such a transformation we mean that every cycle of the new set will be homologous to a sum of cycles of the old, the matrix of the coefficients of the homologies being \pm 1 .

According to the distributive law for indices, if we change fundamental sets the above matrix is merely multiplied to the right or to the left by a matrix of determinant \pm 1 . Therefore the invariant factors of (46.1) are independent of the two fundamental sets, and in fact they are all equal to unity, 
an important result just proved by Veblen, ${ }^{*}$ which we shall also derive later in a new way (No. 65). It follows from this and from Poincaré's theorem on the equality of the numbers $R_{h}, R_{n-h}$, that we may select our sets so that

(a) for $j>R_{h}, \Gamma_{h}^{j}$ and $\Gamma_{n-h}^{j}$ are zero-divisors ;

(b) if $h \neq n / 2$ or if $h=n / 2$ and $n / 2$ is even,

all other indices being zero;

$$
\left(\Gamma_{h}^{i} \cdot \Gamma_{n-h}^{i}\right)=\left\{\begin{array}{l}
+1 \quad \text { if } h \leqq n / 2 \\
(-1)^{(n+1) h} \text { if } h>n / 2
\end{array}\right\} i \leqq R_{h},
$$

(c) if $h=n / 2$ and $n / 2$ is odd, when (46.1) is an alternate matrix,

$$
\left(\Gamma_{n / 2}^{2 i-1} \cdot \Gamma_{n / 2}^{2 i}\right)=-\left(\Gamma_{n / 2}^{2 i} \cdot \Gamma_{n / 2}^{2 i-1}\right)=+1, i \leqq \frac{1}{2} R_{n / 2}
$$

with all other indices again zero. In this case, of course, since the matrix is alternate and of rank $R_{n / 2}$ this last integer is necessarily even, a generalization of the well known result for two-dimensional manifolds.

The possibility of choosing the sets as above is based upon well known theorems on the reduction of matrices with integer terms. $\dagger$

Fundamental sets of the type just described will be called canonical.

\section{Part II. Transformation of Manifolds}

\section{\$1. Product complexes $\ddagger$}

47. Let $E_{p}, E_{q}$ be two cells, $A$ a point of $E_{p}$ or of its boundary, $B$ a similar point for $E_{q}$. Consider the set of couples $A, B$ which by definition vary continuously if either $A$ or $B$ so varies. I say that the set is an $E_{n}, n=p+q$, plus its boundary. This follows at once from the fact that if $x_{1}, x_{2}, \cdots, x_{n}$ are cartesian coördinates for an $S_{n}, E_{p}, E_{q}$ with their boundaries, then the sets in question are respectively homeomorphic to the following three sets:

$$
\begin{aligned}
& 0 \leqq x_{i} \leqq 1, \quad i \leqq p ; \quad x_{p+i}=0 ; \\
& x_{i}=0, i \leqq p ; \quad 0 \leqq x_{p+j} \leqq 1 ; \\
& 0 \leqq x_{i} \leqq 1, \quad i=1,2, \cdot . \cdot, n .
\end{aligned}
$$

* These Transactions, vol. 25 (1923), p. 540. See in the same connection my Monograph already quoted, p. 13. In two recent notes of the Proceedings of the $\mathrm{National}$ Academy of Sciences, vol. 10 (1924), pp. 99-103, J. W. Alexander generalizing this notion has been led to a new set of topological invariants.

† See the expository paper by Veblen and Franklin in the Annals of Mathematics, ser. 2, vol. 23.

¥ The term and corresponding notation are due to E. Steinitz, Sitzungsberichte der Berliner mathematischen Gesellschaft, vol 7 (1908). See also in this connection H. Tietze, Abhandlungen des mathematischen Seminars zu Hamburg, vol. 2 (1923), p. 37, H. Kunneth, Mathematische Annalen, vol. 90 (1923), p. 65, and my paper in these Transactions, vol. 22 (1921), p. 362. 
48. Let $\Pi_{p}, \Pi_{q}$ be polyhedra in spaces $S_{r}, S_{t}$, by means of which straightness and distances are defined for $E_{p}$ and $E_{q}$ and let $x_{1}, \cdots, x_{r}$ and $y_{1}, \cdots, y_{t}$ be cartesian coördinates for $S_{r}$ and $S_{t}$. We may refer an $S_{r+t}$ to the set of cartesian coördinates $x_{i}, y_{j}$. Then if a point $(x)$ describes $\Pi_{p}$ and a point $(y)$ describes $\Pi_{q}$ the corresponding point $(x, y)$ of $S_{r+1}$ describes a $\Pi_{n}$ homeomorphic to $E_{n}$ plus its boundary. The geometry of this is very elementary and we leave it to the reader. This $\Pi_{n}$ shall be chosen as basis for straightness and distances on $E_{n}$ and its boundary.

The point $A, B$ of $E_{n}$ or its boundary shall be designated henceforth by $A \times B$. Let $A A_{1} \cdots A_{p}, B B_{1} \cdots B_{q}$ be indicatrices of $E_{p}$ and $E_{q}$. We shall agree to sense $E_{n}$ by the indicatrix

$$
A \times B A_{1} \times B \cdot \cdot A_{p} \times B A \times B_{1} \cdot \cdot A \times B_{q},
$$

and the cell so sensed shall be denoted by $E_{p} \times E_{q}$ and called product of the two cells, its factors. ${ }^{*}$ The notation $A \times B$ merely corresponds to $p=q=0$. At once, we have

$$
E_{p} \times E_{q}=-\left(-E_{p}\right) \times E_{q}=-E_{p} \times\left(-E_{q}\right)=(-1)^{p q} E_{q} \times E_{p} .
$$

Let $a_{p-1}^{i}, l_{q-1}^{j}$ be the boundary cells of $E_{p}$ and $E_{q}$, so sensed that

$$
E_{p} \equiv \sum a_{p-1}^{i}, \quad E_{q} \equiv \sum b_{q-1}^{i}
$$

Then

$$
E_{p} \times E_{q} \equiv \sum \epsilon_{i} a_{p-1}^{i} \times E_{q}+\sum \eta_{j} E_{p} \times b_{q-1}^{j},
$$

where $\epsilon_{i}$ and $\eta_{i}$ are \pm 1 . To determine their actual value assume that $B B_{1} \cdots B_{q-1}$ is on $b_{q-1}^{j}$. Then $(-1)^{q} \cdot B B_{1} \cdots B_{q-1}$ is an indicatrix of $b_{q-1}^{j}$ as boundary cell of $E_{q}$. That of $E_{p} \times b_{q-1}^{j}$ is

$$
(-1)^{q} A \times B A_{1} \times B \cdots A_{p} \times B A \times B_{1} \cdots A \times B_{q-1} \text {. }
$$

Comparing with the indicatrix of $E_{p} \times E_{q}$ we find $\eta_{j}=(-1)^{q}$. If we interchange $E_{p}$ and $E_{q}$ we shall find by applying this very relation $(-1)^{q} \epsilon_{i}=$ $(-1)^{q}$, hence $\epsilon_{i}=1$, so that finally $(48.2)$ becomes

$$
E_{p} \times E_{q} \equiv \sum a_{p-1}^{i} \times E_{q}+(-1)^{p} \sum E_{p} \times b_{q-1}^{i},
$$

which describes the boundary of the product.

49. Let now $C_{p}, C_{q}$ be any complexes, $a_{p}^{s}$ a generic $p$-cell of the first, $b_{a}^{j}$ one of the second. We define their product by the relation

$$
C_{p} \times C_{q}=\sum a_{p}^{i} \times b_{q}^{i} .
$$

\footnotetext{
- Concerning this symbolic product see the footnote to No. 7.
} 
By applying (48.3) to each term at the right we find

$$
C_{p} \times C_{q} \equiv \sum a_{p-1}^{i} \times b_{q}^{j}+(-1)^{p} \sum a_{p}^{i} \times b_{q-1}^{j}
$$

where only the boundary $(p-1)$ - and $(q-1)$-cells appear at the right with the very orientation that they possess in the boundary congruences of the complexes. Hence if $C_{p-1}$ and $C_{q-1}$ are these boundaries,

$$
C_{p} \times C_{q} \equiv C_{p-1} \times C_{q}+(-1)^{p} C_{p} \times C_{q-1}
$$

From (49.2) we have the following:

I. The product is orientable (in the sense that a manifold is) if and only if each factor is.

II. The product of two manifolds is a manifold.

III. The product of two complexes is without boundary if, and only if, the complexes themselves have none. Observe that all this can be extended to singular complexes, hence

IV. The product of two cycles of the factors is a cycle of the product.

From polyhedra of the factors we derive as in No. 48 one for the product, hence corresponding definitions of straightness and distances.

50. Every $\Gamma_{k}$ of $C_{p} \times C_{q}$ is homologous to a polyhedral cycle $\Gamma_{k}{ }^{\prime}$ (Coll. Lect., p. 120). Let $a$ be a point of the cell $a_{p}^{l} \times b_{q}^{j}$ not on $\Gamma_{k}{ }^{\prime}$. Draw a rectilinear segment from $a$ to every point $\beta$ of $\Gamma_{k}{ }^{\prime}$ on the cell, to its intersection at $\gamma$ with the boundary. The set of all segments $\beta \gamma$ constitutes a polyhedral $C_{k+1}$. The effect of subtracting its boundary from $\Gamma_{k}{ }^{\prime}$ is to reduce the latter to a similar cycle without any points on $a_{p}^{i} \times b_{a}^{j}$. On proceeding thus with all such cells, then with the $C_{n-1}$ made up with the cells of less than $n$ dimensions of $C_{p} \times C_{q}$, and so on, we shall reduce $\Gamma_{k}{ }^{\prime}$ to a homologous polyhedral cycle whose points are all on cells of at most $k$ dimensions of the complex. From the theorem of the Colloquium Lectures just recalled, as applied to the $C_{k}$ made up of the cells of at most $k$ dimensions of our complex, follows that the reduced cycle, which we still call $\Gamma_{k}{ }^{\prime}$, is a sum of $k$-cells of $C_{p} \times C_{q}$. This may also be shown by remarking that if it has points on an $E_{k}$ the whole cell must belong to it, else it would have a boundary on it.

We conclude then that every cycle of a product is homologous to a sum of products of cells of its two factors.

Suppose that such a $\Gamma_{k}$ bounds on $C_{p} \times C_{q}$. We may apply to the complex that it bounds the identical reasoning, the operations made not affecting the boundary, and we conclude that if a cycle, sum of products of cells of the factors, bounds on the product, it bounds a complex which is also such a sum. 
Henceforth we shall use the notation $\gamma_{k}, \delta_{k}$ for the $k$-cycles of $C_{p}$ and $C_{q}$, keeping $\Gamma_{k}$ itself for those of the product $C_{p} \times C_{q}$.

51. As shown by Veblen* every $E_{k}$ of $C_{p}$ is expressible as a sum of multiples of certain cycles $\gamma_{p}^{*}$ and certain cells $a_{k}^{j}$. Of course no sum of these particular $a$ 's is a $\gamma$, hence every $\gamma_{k}$ of $C_{p}$ is a sum of multiples of the cycles $\gamma_{k}^{j}$ alone. It is not ruled out that some of the latter bound, but their set may be so chosen as to include a jundamental set for the $k$-cycles. There exists of course a similar set $\delta_{\boldsymbol{k}}^{j}, b_{\boldsymbol{k}}^{j}$ for $C_{q}$.

52. THEOREM. The k-cycles which are products of cycles taken from fundamental sets of the factors constitute a fundamental set for the product.

According to No. 50, for any $\Gamma_{k}$ we have

$$
\Gamma_{k} \sim \sum \gamma_{\mu}^{i} \times \delta_{k-\mu}^{j}+\sum \gamma_{\mu}^{i} \times b_{k-\mu}^{i}+\sum a_{\mu}^{i} \times \delta_{k-\mu}^{j}+\sum a_{\mu}^{i} \times b_{k-\mu}^{i} .
$$

We must express the fact that the right side has no boundary. The boundary of $a_{\mu}^{i} \times b_{k-\mu}^{j}$ is a sum of terms $\gamma_{\mu-1} \times b_{k-\mu}^{j}, a_{\mu} \times \delta_{k-\mu-1}$ where $\gamma_{\mu-1}, \delta_{k-\mu-1}$ are sums of cycles described in the preceding number. The boundaries of an $a \times \delta$ or of a $\gamma \times b$ are both of type $\gamma \times \delta$. Hence the boundaries of the terms in the fourth sum can only cancel each other, which compels the sum to be similar to the third. Hence for the cycle we have an homology

$$
\Gamma_{k} \sim \sum \gamma_{\mu}^{i} \times \delta_{k-\mu}^{i}+\sum \gamma_{\mu}^{i} \times b_{k-\mu}^{j}+\sum a_{\mu}^{i} \times \delta_{k-\mu}^{j} \text {. }
$$

We may assume that the second sum cannot be split into two, one of which is a cycle, for it would then be of the same type as the first and could be merged with it. Similarly for the third sum. From this follows that if the second sum is absent so is the third and conversely. Also since the boundaries of the terms in the last two sums must cancel each other, any term $\gamma_{\mu}^{i} \times b_{k-\mu}^{j}$ in the second sum contains only such a $\gamma$ as may come from the boundaries of terms in the third. $\gamma_{\mu}^{i}$ bounds then a certain $C_{\mu+1}$ of $C_{p}$. Let

$$
b_{k-\mu}^{i} \equiv \sum \epsilon_{j \delta} \delta_{k-\mu-1}^{0}
$$

At once,

$$
C_{\mu+1} \times b_{k-\mu}^{j} \equiv \gamma_{\mu}^{i} \times b_{k-\mu}^{j}+(-1)^{\mu+1} \sum \epsilon_{j o} C_{\mu+1} \times \delta_{k-\mu-1}^{*} \sim 0 \quad\left(\bmod C_{p} \times C_{p}\right)
$$

On subtracting this bounding cycle from the second expression for $\Gamma_{k}$ the term $\gamma_{\mu}^{i} \times b_{k-\mu}^{j}$ will have been replaced by a sum of terms that will go

- Coll. Lect., p. 116. The result in question, not stated explicitly by him, is really derived in the course of the discussion which leads to the theorem there stated. 
into the first or the third sum. Therefore by a previous remark, the third must then also disappear. Hence

$$
\Gamma_{k} \sim \sum \gamma_{\mu}^{i} \times \delta_{k-\mu}^{i}
$$

If $\gamma_{\mu}^{i}$ bounds $C_{\mu+1}$ of $C_{p}$, then $\gamma_{\mu}^{i} \times \delta_{k-\mu}^{j}$ bounds $C_{\mu+1} \times \delta_{k-\mu}^{j}$; hence in the expression of $\Gamma_{k}$ only the terms for which neither $\gamma$ nor $\delta$ bound need to be preserved, which proves our theorem.

It is not difficult to show that if two factor cycles do not bound their product does not bound, but as this is unnecessary for the sequel we omit the proof. A corollary is that no cycle of the fundamental sets obtained bounds on $C_{p} \times C_{q}$.

Of more import is the following observation. If $\gamma_{\mu}$ is a zero-divisor for $C_{p}, \gamma_{\mu} \times \delta_{k-\mu}$ is a zero-divisor or else bounds and similarly with $\delta$ in place of $\gamma$. For if $t \gamma_{\mu}$ bounds $C_{\mu_{+1}},\left(t \gamma_{\mu}\right) \times \delta_{k-\mu}$ bounds $C_{\mu_{+1}} \times \delta_{k-\mu}$. Hence the theorem that we have proved holds even when fundamental sets with respect to the operation $\approx$ take the place of the others. For we may now eliminate from the fundamental sets of the product all cycles of which a factor is a zero-divisor.*

53. Product of orientable manifolds. We know already (No. 49) that if $M_{p}, M_{q}$ are orientable manifolds so is $M_{p} \times M_{q}$. We are now especially concerned with the question of Kronecker indices.

Let $E_{h}, E_{p-h}$ be simplicial cells on $M_{p}$ and suppose that they intersect at $A$. Let $E_{k}^{\prime}, E_{q-k}^{\prime}$ and $B$ be similar elements for $M_{q}$. Then $E_{h} \times E_{k}{ }^{\prime}$ and $E_{p-\Lambda} \times E_{q-k}^{\prime}$ intersect at $A \times B$ on $M_{p} \times M_{q}$ and the question is to determine their index in terms of $\left(E_{h} \cdot E_{p-k}\right)$, and $\left(E_{k}^{\prime} \cdot E_{q-k}^{\prime}\right)$.

Let the $A$ 's and $B$ 's of No. 48 now serve to determine indicatrices for the manifolds, as previously for the cells. As a matter of fact, the $M$ 's might simply be $E$ 's without changing anything.

We may now assume that the indicatrices are

$$
\begin{array}{ll}
A A_{1} \cdot \cdot A_{h}, & A A_{k+1} \cdot \cdot A_{p} \text { for } E_{h} \text { and } E_{p-h}, \\
B B_{1} \cdot \cdot B_{k}, & B B_{k+1} \cdot \cdot B_{q} \text { for } E_{k} \text { and } E_{q-k} \cdot
\end{array}
$$

Hence for the above named two-cell products the indicatrices

$$
\begin{aligned}
& A \times B A_{1} \times B \cdots \cdot A_{k} \times B A \times B_{1} \cdot \cdot A \times B_{k}, \\
& A \times B A_{k+1} \times B \cdots A_{p} \times B A \times B_{k+1} \cdot A \times B_{k} .
\end{aligned}
$$

\footnotetext{
* From this follow the results of my Transactions paper and those of Künneth, concerning connectivity and torsion indices.
} 
By comparing with the indicatrix of $M_{p} \times M_{q}$ and applying the rule for the determination of the index we obtain the following formula :

$$
\left(E_{h} \times E_{k}^{\prime} \cdot E_{p-h} \times E_{q-k}^{\prime}\right)=(-1)^{k(p-h)}\left(E_{h} \cdot E_{p-h}\right)\left(E_{k}^{\prime} \cdot E_{q-k}^{\prime}\right) .
$$

It holds even for $k=0, h=p$, when it yields the following result, verifiable directly with ease :

$$
\left(E_{p} \times B \cdot A \times E_{q}\right)=+1 \text {. }
$$

The product $E_{p} \times B$ simply denotes an $E_{p}$ on the product cell, and similarly for $A \times E_{q}$.

Let now $C_{h}, C_{p-h}$ be complexes on $M_{p}$ whose points of intersection are neither on their boundaries nor on that of the manifold, and let $C_{k}^{\prime}, C_{q-k}^{\prime}$ be analogous for $M_{q}$. Polyhedral approximations to $C_{h}$ and $C_{k}{ }^{\prime}$ have for product such an approximation to $C_{h} \times C_{k}{ }^{\prime}$, and similarly for the other two complexes. If the approximations to $C_{h}$ and $C_{p-h}$ and those to $C_{k}^{\prime}$ and $C_{a-k}^{\prime}$ intersect at isolated ordinary points of the complexes, the approximations to the products will behave likewise. Hence from the definition of the index of two polyhedral complexes with well defined intersections, the extension to arbitrary complexes, and (53.1), (53.2), we have

$$
\begin{aligned}
\left(C_{h} \times C_{k}^{\prime} \cdot C_{p-h} \times C_{q-k}^{\prime}\right) & =(-1)^{k(p-h)}\left(C_{h} \cdot C_{p-h}\right)\left(C_{k}^{\prime} \cdot C_{q-k}^{\prime}\right) ; \\
\left(M_{p} \times B \cdot A \times M_{q}\right) & =+1 .
\end{aligned}
$$

The products in (53.4) represent complexes homeomorphic to $M_{p}$ or $M_{q}$ on $M_{p} \times M_{q}$. The approximations to $A$ and $B$ in that case consist in choosing points of their vicinity situated on $p$ - and $q$-cells of the covering complexes of the manifolds which serve to define straightness and distances on them.

Two complexes $C_{h}, C_{p-h-i}$ on $M_{p}$, without points on its boundary, may always be approximated by two that do not intersect. Hence if $C_{k}^{\prime}$, $C_{q-k+i}^{\prime}$ are on $M_{q}$, without points on its boundary, there are non-intersecting polyhedral approximations to $C_{h} \times C_{k}{ }^{\prime}$ and $C_{p-h-i} \times C_{q-k+i}^{\prime}$. Therefore

$$
\left(C_{h} \times C_{k}^{\prime} \cdot C_{p-h-i} \times C_{q-k+i}\right)=0 .
$$

54. TheOREM. Let $M_{p}, M_{q}$ be without boundary and let $\gamma_{k}^{1}, \delta_{k}^{j}$ be the cycles of their canonical fundamental sets. Then except for the signs their products constitute such a set for $M_{p} \times M_{q}$. 
This follows at once from the relations derived from (53.3), (53.4) (53.5) :

$$
\begin{array}{rlr}
\left(\gamma_{\lambda}^{i} \times \delta_{k-\lambda}^{j} \cdot \gamma_{p-\lambda}^{i} \times \delta_{q-k+\lambda}^{j}\right)=(-1)^{(k-\lambda)(p-\lambda)} \cdot\left(\gamma_{\lambda}^{i} \cdot \gamma_{p-\lambda}^{j}\right)\left(\delta_{k-\lambda}^{i} \cdot \delta_{q-k+\lambda}^{j}\right) & \\
\left(\gamma_{\lambda}^{i} \times \delta_{k-\lambda}^{j} \cdot \gamma_{p-\mu}^{i} \times \delta_{q-k+\mu}^{j}\right)=0, & \lambda \neq \mu
\end{array}
$$

They indicate also the manner in which the cycles are to be associated.

Of great importance for transformations is the special case $p=q=k=n$. We have then

$$
\left(\gamma_{\lambda}^{i} \times \delta_{n-\lambda}^{j} \cdot \gamma_{n-\lambda}^{i} \times \delta_{\lambda}^{j}\right)=(-1)^{n(\lambda+1)} \cdot\left(\gamma_{\lambda}^{i} \cdot \gamma_{n-\lambda}^{i}\right) \cdot\left(\delta_{\lambda}^{i} \cdot \delta_{n-\lambda}^{i}\right)
$$

and all other indices vanish.

55. Formulas (53.3), (53.4) are special cases of a more general one corresponding to cycles that are intersections of complexes, which is derived by similar considerations. As we shall not use it later we merely give it here without proof. If $C_{l}$ and $C_{\lambda}$ of $M_{p}$ do not intersect each other's boundary, and have no intersection on the boundary of the manifold, if also $C_{m}^{\prime}, C_{\mu}^{\prime}$ behave likewise relatively to $M_{q}$, the formula in question is as follows :

$$
C_{l} \times C_{m}^{\prime} \cdot C_{\lambda} \times C_{\mu}^{\prime} \sim(-1)^{(p-l)(q-\mu)} \cdot C_{l} \cdot C_{\lambda} \times C_{m}^{\prime} \cdot C_{\mu}^{\prime} \quad\left(\bmod M_{p} \times M_{q}\right) .
$$

\section{§2. TRANSFORMATIONS OF A MANIFOLD WITHOUT BOUNDARY}

56. Let $M_{n}$ be the manifold, $M_{n}^{\prime}$ another copy of it, $T$ a transformation of $M_{n}$ into itself or part of itself, which we subject to this sole condition of a very general nature: If $A$ is any point of $M_{n}, B$ the image of any transform $A^{\prime}$ of it on $M_{n}{ }^{\prime}$, the set of all points $A \times B$ is an $n$-cycle $\Gamma_{n}$ on $M_{n} \times M_{n}{ }^{\prime}$. The inclusiveness of the class of transformations so defined becomes apparent when we remark that all continuous one-valued transformations (for each $A$ only one $A^{\prime}$ varying continuously with $A$ ) belong to it. The non-singular $C_{n}$ without boundary of which $\Gamma_{n}$ is then the image in the sense of No. 6 is $M_{n}$ itself. More generally $k$-valued continuous transformations are also of our type; the corresponding $C_{n}$ is then $k M_{n}$.

Much of the rest of this paper will center around the determination of certain Kronecker indices and it becomes essential to define all orientations involved. Let $C_{n}$ cover $M_{n}$ and let $C_{n}{ }^{\prime}$ be its image covering $M_{n}{ }^{\prime}$. Suppose that $\Pi_{n}$ is a polyhedron which associated with $C_{n}$ serves to define straightness and distances on $M_{n}$. The polyhedron $\Pi_{n}$ has the same cell structure as $C_{n}{ }^{\prime}$ and we shall agree to use it, associated with $C_{n}{ }^{\prime}$, to define straightness 
and distances on $M_{n}{ }^{\prime}$. Then any rectilinear segment of $M_{n}$ has for image a similar one on $M_{n}{ }^{\prime}$ and both have the same length. To an indicatrix $E_{n}$ on $M_{n}$ will correspond an $E_{n}{ }^{\prime}$ on $M_{n}{ }^{\prime}$. We shall name the vertices of $E_{n}{ }^{\prime}$ in the same order as the corresponding vertices of $E_{n}$, and use the simplex so sensed as indicatrix for $M_{n}{ }^{\prime}$. In accordance with our previous conventions the orientation of $M_{n} \times M_{n}{ }^{\prime}$ is now perfectly determined. Owing to its importance for the sequel, it is well perhaps to characterize it more geometrically. Through any point $A \times B$ of the manifold there pass $M_{n} \times B$ and $A \times M_{n}{ }^{\prime}$. Let $B$ be the image of $A$. To $E_{n}$ with $A$ as its first vertex corresponds $E_{n}{ }^{\prime}$ with $B$ as its first vertex. Let $\bar{E}_{n}$ be the image of the first on $M_{n} \times B, \bar{E}_{n}{ }^{\prime}$ that of the second on $A \times M_{n}{ }^{\prime}$. The $E_{2 n}$ indicatrix of the product manifold has its vertices named in the following order: $A \times B$, the other vertices of $\bar{E}_{n}$, the other vertices of $\bar{E}_{n}{ }^{\prime}$.

There remains the orienting of $\Gamma_{n}$. If one cycle is suitable for $T$ so is its opposite. Of the two we shall select the one such that

$$
\left(\Gamma_{n} \cdot A \times M_{n}{ }^{\prime}\right)=a_{0} \geqq 0,
$$

a perfectly definite condition since the integer is a Kronecker index of cycles.

57. Let us apply to $M_{n}$ and $M_{n}{ }^{\prime}$ the very notations of No. 54, so that $\delta_{k}^{\prime}$ is now the cycle of $M_{n}{ }^{\prime}$ that corresponds to $\gamma_{k}^{3}$ on $M_{n}$. We shall then have

$$
\Gamma_{n} \sim \sum \epsilon_{\mu}^{i j} \cdot \gamma_{\mu}^{i} \times \delta_{n-\mu}^{i} \quad\left(\bmod M_{n} \times M_{n}{ }^{\prime}\right) .
$$

The $\gamma$ 's and $\delta$ 's are, it will be recalled, cycles of fundamental sets. In particular $\gamma_{0}, \delta_{0}$ are merely points of $M_{n}, M_{n}{ }^{\prime}$ while $\gamma_{n}, \delta_{n}$ are the manifolds themselves. The number of cycles of the fundamental sets is one for these two extreme cases.

The $\epsilon$ 's are important characteristic integers of $T$. It will be remembered that two transformations are said to be of the same class if they belong to one and the same continuous family of transformations. Whenever $T$ varies within its class, $\Gamma_{n}$ is continuously deformed (a condition that might serve to define the class) and $\Gamma_{n}$ remains homologous to a fixed cycle, so that the $\epsilon$ 's are unchanged. Hence to a given class of transformations corresponds a fixed set of $\epsilon$ 's. Whether the converse holds or not is as yet unknown.

58. We must now introduce the notion of the transform of a cycle of $M_{\text {n }}$ by $T$. The necessity of defining such a notion with precision arises from the fact that while $T$ is a point transformation a cycle is not a mere point set, but consists of a set of cells each taken with a certain multiplicity. The cycle is then really a symbol attached to a set of cells, and what is meant by its transform is by no means evident a priori. 
Let $\gamma_{\mu}, 0 \leqq \mu \leqq n$, be any cycle of $M_{n}$. If it is not polyhedral we approximate it by one that is and that furthermore has been reduced in accordance with the theorem of No. 23. Then $\gamma_{\mu} \times M_{n}{ }^{\prime}$ behaves likewise and we may also approximate $\Gamma_{n}$ in the same manner so that the two have a well defined intersection $\Gamma_{n}, \gamma_{\mu} \times M_{n}{ }^{\prime}=\Gamma_{\mu}$. (We economize in notations by designating the approximations like the cycles themselves.) To every point $A \times B$ of $\Gamma_{\mu}$ corresponds a unique $B$ on $M_{n}{ }^{\prime}$ varying continuously when $A \times B$ so varies on $\Gamma_{\mu}$. Hence $B$ gives rise to a cycle $\bar{\delta}_{\mu}$ on $M_{n}{ }^{\prime}$. It is a singular complex of $M_{n}^{\prime}$, continuous image of $\Gamma_{\mu}$ in the sense of No. 6 . The image $\bar{\gamma}_{\mu}$ of $\bar{\delta}_{\mu}$ on $M_{n}$ is by definition the transform of $\gamma_{\mu}$ by $T$. It will be observed that $\bar{\delta}_{\mu}$ is polyhedral, hence so is $\bar{\gamma}_{\mu}$. For $\bar{\delta}_{\mu}$ is represented on the copy $A \times M_{n}{ }^{\prime}$ of $M_{n}{ }^{\prime}$ by the cycle $A \times \bar{\delta}_{\mu}$ which is the locus of all intersections of $A \times M_{n}{ }^{\prime}$ with all manifolds $M_{n} \times B$ that meet $\Gamma_{n} . \gamma_{\mu} \times M_{n}{ }^{\prime}$. In this fashion, however, $A \times \bar{\delta}_{\mu}$ appears as an intersection of polyhedral complexes, and it is therefore also polyhedral. If $A \times B A_{1} \times B_{1} \cdots A_{\mu} \times B_{\mu}$ is an indicatrix of $\Gamma_{\mu}$ then $B B_{1} \cdots B_{\mu}$ is an indicatrix of $\bar{\delta}_{\mu}$.

The preceding definition is justified by the fact that for $\mu=0$, when $\gamma_{0}$ consists of a finite number of points, the correct transforms of these points are obtained. Furthermore (No. 62) when $T$ is the identity all cycles are left invariant, which is as it should be with any properly selected definition.

Let the cycle transformations be represented by homologies

$$
\bar{\gamma}_{\mu}^{i} \sim \sum a_{\mu}^{i j} \gamma_{\mu}^{i j}
$$

$\left(\bmod M_{n}\right)$.

For any given $T$ one is far more likely to possess information concerning the $a$ 's than concerning the $\epsilon$ 's. Hence the interesting and important problem arises to determine the e's in terms of the a's. This problem shall be solved partly in a more narrow form. Let us drop zero divisors throughout. In accordance with No. 52, with the $R$ 's denoting the connectivity indices of $M_{n}$, and assuming that among the cycles $\gamma_{\mu}^{\prime}$ the first $R_{\mu}$ are independent, we shall now have in place of (57.1) and (58.1), the following relations whose appearance is the same:

$$
\begin{array}{lr}
\Gamma_{h} \approx \sum \epsilon_{\mu}^{i j} \cdot \gamma_{\mu}^{i} \times \delta_{n-\mu}^{j} & \left(\bmod M_{n} \times M_{n}^{\prime}\right) ; \\
\bar{\gamma}_{\mu}^{i} \approx \sum a_{\mu}^{i_{j}} \gamma_{\mu}^{i} & \left(\bmod M_{n}\right),
\end{array}
$$

where as before $\mu$ runs from 0 to $n$, but, for each $\mu, i$ and $j$ run from 1 to $R_{\mu}=R_{n-\mu}$. The problem that we shall completely solve is the determination of the e's within this range in terms of the a's similarly limited. This will suffice to provide all that is needed in our applications to fixed points and coincidences. 
59. An important formula. The solution of our problem is based upon the following formula, whose proof will now occupy us:

$$
\left(\Gamma_{n} \cdot \gamma_{\mu} \times \delta_{n-\mu}\right)=(-1)^{\mu} \cdot\left(\bar{\gamma}_{\mu} \cdot \gamma_{n-\mu}\right)
$$

with indices computed at the left as to $M_{n} \times M_{n}{ }^{\prime}$ and at the right as to $M_{n}$. In place of (59.1) it will be found more convenient to prove the equivalent

$$
\left(\Gamma_{n} \cdot \gamma_{\mu} \times \delta_{n-\mu}\right)=(-1)^{\mu} \cdot\left(\dot{\delta}_{\mu} \cdot \delta_{n-\mu}\right),
$$

where the last index is computed as to $M_{n}{ }^{\prime}$, and to this we now turn our attention.

60. By means of our usual approximations as applied to $\Gamma_{n}, \gamma_{\mu}$ and $\delta_{n-\mu}$, we may so arrange matters that all cycles are polyhedral and have well defined intersections. Let $A \times B$ be an intersection of $\Gamma_{n}$ with $\gamma_{\mu} \times \delta_{n-\mu}$. Then $B$ is an intersection of $\bar{\delta}_{\mu}$ with $\delta_{n-\mu}$. Therefore we need only to show that the contributions of the two points to their respective indices have the ratio $(-1)^{\mu}$. Owing to the distributive law we may assume that all complexes are cells and that the points count for \pm 1 in the indices, or what is the same, that the manifolds are linear spaces and $T$ a projectivity. The method of matrices (No. 4) will be found most convenient for our purpose.

61. We begin with the contribution of $A \times B$ to the left side of (59.2). Let $A, B$, and $A \times B$ be origins of cartesian axes for the spaces $M_{n}, M_{n}{ }^{\prime}$, and their product, the coördinates being $x_{1}, \cdots, x_{n}$ for $M_{n}, y_{1}, \cdots, y_{n}$ for $M_{n}{ }^{\prime}, x_{1}, \cdots, x_{n}, y_{1}, \cdots, y_{n}$ for $M_{n} \times M_{n}{ }^{\prime}$. To the points $(x),(y)$ of $M_{n}, M_{n}^{\prime}$ corresponds the point $(x, y)$ of the product.

We can assume for $M_{n}$ a matrix-indicatrix in the sense of No. 4, of type

$$
\left\|\begin{array}{lll}
X_{\mu} & , & 0 \\
0 & , & X_{n-\mu}
\end{array}\right\|
$$

where the $X$ 's denote square matrices of order equal to the index with determinants equal to +1 , and the zeros matrices whose terms all vanish. The meaning of (61.1) is clear. If $A_{i}$ is the point of $M_{n}$ whose coördinates are the terms in the $i$ th row, then $A A_{1} \cdots A_{n}$ is an indicatrix of $M_{n}$. We may so select it that $A A_{1} \cdots A_{\mu}$ and $A A_{\mu+1} \cdots A_{n}$ are indicatrices of $\gamma_{\mu}$ and $\gamma_{n-\mu}$. This implies a definite orientation for them, but if we invert $\gamma_{\mu}$ or $\gamma_{n-\mu}$ we also invert $\bar{\delta}_{\mu}$ or $\delta_{n-\mu}$ and therefore (59.2) is unaffected, so that there is really no restriction. In the same sense as for $M_{n}$, we may say that the matrices

$$
\left\|X_{\mu}, 0\right\|,\left\|0, X_{n-\mu}\right\|
$$

define indicatrices of $\gamma_{\mu}$ and $\gamma_{n-\mu}$. 
To the images of the transforms of $M_{n}, \gamma_{\mu}, \gamma_{n-\mu}$, by $T$, on $M_{n}{ }^{\prime}$, correspond matrix-indicatrices

$$
\left\|\begin{array}{l}
Y_{\mu}, Y \\
Y^{\prime}, Y_{n-\mu}
\end{array}\right\|,\left\|Y_{\mu}, Y\right\|,\left\|Y^{\prime}, Y_{n-\mu}\right\|
$$

where $Y, Y^{\prime}$ are rectangular arrays and the two other $Y^{\prime}$ 's square arrays of order equal to their index. The first corresponds to $\pm M_{n}{ }^{\prime}=\epsilon M_{n}{ }^{\prime}$, where the sign is plus if $T$ maintains the indicatrix on $M_{n}$, minus if it inverts it. The second and third matrices correspond to $\bar{\delta}_{\mu}$ and $\delta_{n-\mu}$. Since (61.1) defines an indicatrix of $M_{n}{ }^{\prime}$ when each row is considered as the coördinates of a $y$ point, and since its determinant, product of $\left|X_{\mu}\right|$ and $\left|X_{n-\mu}\right|$, is $+1, \epsilon$ has the sign of the determinant

$$
\left|\begin{array}{lll}
Y_{\mu} & , & Y \\
Y^{\prime} & , & Y_{n-\mu}
\end{array}\right|
$$

From the definition of $\Gamma_{n}$ we conclude that

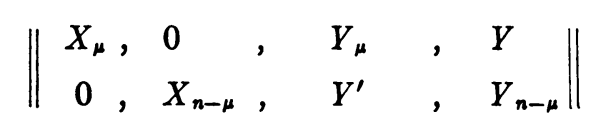

in a matrix-indicatrix of $\theta \Gamma_{n}, \theta= \pm 1$. To determine the sign of $\theta$ observe that since $A \times M_{n}{ }^{\prime}$ has for matrix-indicatrix

$$
\left\|\begin{array}{ccccccc}
0 & , & 0 \\
0 & , & 0 & , & X_{\mu} & , & 0 \\
& 0
\end{array}\right\|,
$$

the contribution of $A \times B$ to $\left(\theta \Gamma_{n} \cdot A \times M_{n}{ }^{\prime}\right)$ has the sign of the determinant

$$
\left|\begin{array}{lllllll}
X_{\mu} & , & 0 & , & 0 & , & 0 \\
0 & , & X_{n-\mu} & , & 0 & , & 0 \\
0 & , & 0 & , & X_{\mu} & , & 0 \\
0 & , & 0 & , & 0 & , & X_{n-\mu}
\end{array}\right|=+1 .
$$

Hence $\theta$ represents the contribution of the point to the integer $a_{0}=$ $\left(\Gamma_{n} \cdot A \times M_{n}{ }^{\prime}\right)$ of No. 56. For example, in the case of the identical transformation, every point of intersection of the two cycles must contribute +1 , therefore $\theta=+1$. This is also true for a continuous 1 to $n$ transformation, where the contributions to the index have a constant sign. That $\theta$ is not -1 in these two cases comes from the condition $a_{0}>0$ by which we have definitely oriented $\Gamma_{n}$. 
But this is a digression from our main topic to which we now return. It is readily seen that

$$
\left\|\begin{array}{ccccccc}
X_{\mu} & , & 0 & , & 0 & , & 0 \\
0 & , & 0 & , & 0 & , & X_{n-\mu}
\end{array}\right\|
$$

is a matrix-indicatrix for $\gamma_{\mu} \times \delta_{n-\mu}$. As $M_{n} \times M_{n}{ }^{\prime}$ has for matrix-indicatrix

$$
\left\|\begin{array}{lllllll}
X_{\mu} & , & 0 & , & 0 & , & 0 \\
0 & , & X_{n-\mu} & , & 0 & , & 0 \\
0 & , & 0 & , & X_{\mu} & , & 0 \\
0 & , & 0 & , & 0 & , & X_{n-\mu}
\end{array}\right\|
$$

whose determinant is +1 , we conclude from the definition of the Kronecker index that the contribution of $A \times B$ to $\left(\theta \Gamma_{n} \cdot \gamma_{\mu} \times \delta_{n-\mu}\right)$ has the sign of the determinant

$$
\left|\begin{array}{lllllll}
X_{\mu} & , & 0 & , & Y_{\mu} & , & Y \\
0 & , & X_{n-\mu} & , & Y^{\prime} & , & Y_{n-\mu} \\
X_{\mu} & , & 0 & , & 0 & , & 0 \\
0 & , & 0 & , & 0 & , & X_{n-\mu}
\end{array}\right|=(-1)^{\mu}\left|Y_{\mu}\right| .
$$

The contribution of $A \times B$ to $\left(\Gamma_{n} \cdot \gamma_{\mu} \times \delta_{n-\mu}\right)$ has then the sign of $(-1)^{\mu} \theta\left|Y_{\mu}\right|$.

62. Let us now examine the contribution of $B$ to $\left(\bar{\delta}_{\mu} \cdot \delta_{n-\mu}\right)$. Suppose that we have found for $\Gamma_{n} \cdot \gamma_{\mu} \times M_{n}^{\prime}$ a matrix-indicatrix

$$
\left\|Z, Z^{\prime}\right\|
$$

where $Z, Z^{\prime}$ are matrices with $\mu$ rows and $n$ columns. Then $Z^{\prime}$ will define an indicatrix for $\bar{\delta}_{\mu}$, referred of course to the $y$ coördinates. For any row $x_{1}, \cdots, x_{n}, y_{1}, \cdots, y_{n}$ represents a point $A^{\prime} \times B^{\prime}$ such that $B^{\prime}$ is the point of $M_{n}^{\prime}$ with the $y$ coördinates. From this and the remark, No. 58, as to relations between indicatrices on $\Gamma_{n}$ and $\bar{\delta}_{\mu}$, follows the property of $Z^{\prime}$.

Now $\gamma_{\mu} \times\left(\epsilon M_{n}^{\prime}\right)$ has for matrix-indicatrix

$$
\left\|\begin{array}{lllllll}
X_{\mu} & , & 0 & , & 0 & , & 0 \\
0 & , & 0 & , & Y_{\mu} & , & Y \\
0 & , & 0 & , & Y^{\prime} & , & Y_{n-\mu}
\end{array}\right\|
$$

which we may replace by

$$
\left\|\begin{array}{lllllll}
X_{k} & , & 0 \\
0 & , & 0 \\
0 & , & 0 & , & Y_{\mu} & , & Y \\
Y^{\prime} & , & Y_{n-\mu}
\end{array}\right\|,
$$


derived from it by addition of rows, as this change of indicatrix merely corresponds to an affine transformation of determinant +1 applied to the $S_{\mu_{+n}}$ involved, and is therefore permissible. The first row defines now an indicatrix of $\zeta \theta \Gamma_{n} \cdot \gamma_{\mu} \times\left(\epsilon M_{n}{ }^{\prime}\right)$, where $\zeta= \pm 1$. From our mode of defining sensed intersections (No. 12) it follows that $\zeta$ has the same sign as the determinant

$$
\left|\begin{array}{lllllll}
X_{\mu} & , & 0 & , & Y_{\mu} & , & Y \\
0 & , & X_{n-\mu} & , & Y^{\prime} & , & Y_{n-\mu} \\
0 & , & 0 & , & Y_{\mu} & , & Y \\
0 & , & 0 & , & Y^{\prime} & , & Y_{n-\mu}
\end{array}\right|=\left|\begin{array}{lll}
Y_{\mu} & , & Y \\
Y^{\prime} & , & Y_{n-\mu}
\end{array}\right|,
$$

that is, the sign of $\epsilon$. As both are unity in absolute value, $\zeta=\epsilon$, so that actually the matrix

$$
\left\|X_{\mu}, \quad 0 \quad, \quad Y_{\mu}, \quad Y\right\|
$$

defines an indicatrix of $\epsilon \theta \Gamma_{n} \cdot \gamma_{\mu} \times\left(\epsilon M_{n}^{\prime}\right)=\theta \Gamma_{n} \cdot \gamma_{\mu} \times M_{n}^{\prime}$. According to what has been said at the beginning of this number, then

$$
\left\|Y_{\mu}, \quad Y\right\|
$$

defines an indicatrix for $\theta \bar{\delta}_{\mu}$.

Since $M_{n}{ }^{\prime}$ has a matrix-indicatrix of determinant +1 , the contribution of $B$ to $\left(\theta \bar{\delta}_{\mu} \cdot \delta_{n-\mu}\right)$ has the sign of the determinant

$$
\left|\begin{array}{lll}
Y_{\mu} & , & Y \\
0 & , & X_{n-\mu}
\end{array}\right|
$$

since the last line is a matrix-indicatrix for $\delta_{n-\mu}$. As the determinant has for value $\left|Y_{\mu}\right|$, the contribution of $B$ to $\left(\bar{\delta}_{\mu} \cdot \delta_{n-\mu}\right)$ has the same sign as $\theta\left|Y_{\mu}\right|$. Its ratio to that of $\left(\Gamma_{n} \cdot \gamma_{\mu} \times \delta_{n-\mu}\right)$ is then $(-1)^{\mu}$, which suffices. to prove (59.1).

Remark. One incidental result follows readily from our discussion. The indicatrix of $\bar{\delta}_{\mu}$ coincides with the image of the transform of that of $\gamma_{\mu}$ or with its opposite according as $\theta$ is positive or negative. Upon translating this back to $M_{n}$ itself, we find the following result. If $A \times B$ contributes +1 to $\left(\Gamma_{n} \cdot A \times M_{n}^{\prime}\right)$ then the indicatrix of the transform of any cycle through $A$ is the transform of the indicatrix; it is the opposite of it if the contribution is -1 . For example in the case of a continuous sense preserving 1 to $n$ transformation, the indicatrix of the transform is always the transform of the indicatrix. In particular for the identical transformation the two coincide, and $\bar{\gamma}_{\mu}$ not only coincides with $\gamma_{\mu}$ element for element, but also 
with preservation of orientation. This justifies in a sense our convention as to sensing $\Gamma_{n}$ and our definition of the transform of a cycle (No. 58).

63. It is important to emphasize the fact that the extreme values $\mu=0$, $n$ are not exceptional at all. For $\mu=0$, we have already imposed

$$
\left(\Gamma_{n} \cdot A \times M_{n}{ }^{\prime}\right)=a_{0} .
$$

The interpretation given for this integer in No. 60 fits in perfectly with our discussion. Let $a_{n}$ be the similar integer for $T^{-1}$. Its interpretation is then this :* An arbitrary $E_{n}$ of $M_{n}$ is covered with a certain number of cells of $T \cdot M_{n}$. Among these there will be, say, $k^{\prime}$ positive cells, $k^{\prime \prime}$ negative cells of $M_{n}$ and $a_{n}=k^{\prime}-k^{\prime \prime}$. Here of course $a_{n}$ may well be negative. Thus if $T$ is a homeomorphism inverting the indicatrix, $a_{n}=-1$. An example of this is the symmetry of a sphere with respect to a diametral plane. It is important to remember that the $T$ considered is not the initial transformation but one that corresponds to a polyhedral approximation of its $\Gamma_{n}$.

If we compute the index as to $M_{n}{ }^{\prime} \times M_{n}$ we obtain then

$$
\left(\Gamma_{n} \cdot B \times M_{n}\right)=\left(\Gamma_{n} \cdot M_{n} \times B\right)=a_{n} .
$$

If a manifold is inverted all corresponding indices must be changed in sign, an immediate corollary of their definition. As $M_{n} \times M_{n}{ }^{\prime}=(-1)^{n} M_{n}{ }^{\prime} \times M_{n}$, when the index is computed with reference to $M_{n} \times M_{n}{ }^{\prime}$,

$$
\left(\Gamma_{n} \cdot M_{n} \times B\right)=(-1)^{n} a_{n} .
$$

This is what (59.1) becomes for $\mu=n$. Both (63.1) and (63.2) can also be derived with ease by means of matrix-indicatrices. Indeed this would merely involve repeating the discussion of Nos. 60, 61 .

Observe that the notation $a_{0}, a_{n}$ is in accord with the meaning given to $a_{\mu}^{i j}$ in No. 57. They correspond to the homologies describing the behavior of the zero-cycle (a point) and the $n$-cycle $\left(M_{n}\right.$ itself). Explicitly for $\mu=0, n, i$ and $j$ can only be unity, and $a_{0}^{11}=a_{0}, a_{n}^{11}=a_{n}$.

64. We now pass to the actual determination of the $\epsilon$ 's. If we remember that in calculating Kronecker indices zero-divisors may be dropped, we see that in applying (59.1) we may substitute from formulas (58.2) and (58.3) in place of (57.1) and (58.1). Let us then substitute in (59.1) for $\Gamma_{n}$ its expression as given by (58.2), for $\gamma_{\mu}, \delta_{n-\mu}$ the cycles $\gamma_{\mu}^{h}, \delta_{n-\mu}^{k}$, a nd for $\bar{\gamma}_{\mu}^{h}$ its expression (58.3). We obtain

$$
\sum_{i, j=1}^{R_{\mu}} \epsilon_{n-\mu}^{i j}\left(\gamma_{n-\mu}^{i} \times \delta_{\mu}^{i} \cdot \gamma_{\mu}^{h} \times \delta_{n-\mu}^{k}\right)=(-1)^{\mu} \sum_{j=1}^{R_{\mu}} a_{\mu}^{h_{\mu}}\left(\gamma_{\mu}^{i} \cdot \gamma_{n-\mu}^{k}\right),
$$

* This is the same as Brouwer's degree of $T$. See Mathematische Annalen, vol. 71 (1911), p. 105. He limits himself to the case where every point has a unique transform, which is a special case of transformations with $a_{0}=1$. 
all other terms having dropped out in accordance with (54.1). Transforming the left side by means of (53.3) and (53.4) this becomes

$$
(-1)^{\mu(n+1)} \sum_{i, j=1}^{R_{\mu}} i_{\epsilon_{-\mu}}^{i j}\left(\gamma_{\mu}^{k} \cdot \gamma_{n-\mu}^{j}\right)\left(\gamma_{\mu}^{j} \cdot \gamma_{n-\mu}^{k}\right)=\sum_{j=1}^{R_{\mu}} a_{\mu}^{n j}\left(\gamma_{\mu}^{j} \cdot \gamma_{n-\mu}^{k}\right) .
$$

For each $\mu$ we have here a set of $R_{\mu}^{2}$ linear equations in the $B_{\mu}$ unknowns $\epsilon_{n-\mu}^{i j}$. The determinant of their coefficients is a power of the determinant

$$
\left|\left(\gamma_{\mu}^{i} \cdot \gamma_{n-\mu}^{i}\right)\right|,
$$

whose value is actually \pm 1 , as may be deduced from Veblen's theorem mentioned in Part I, §6. These equations may therefore be solved even explicitly if need be, a task which presents little interest. However, if, as we shall assume from now on, the fundamental sets on $M_{n}$ are canonical the solution can be carried through in an instant.

For if we substitute this time the indices as given in Part I, $\S 6$, and as they must be applied here, we have at once

(a) $\mu \neq \frac{1}{2} n$ or $=\frac{1}{2} n=$ an even integer.

Then

$$
\begin{aligned}
& \stackrel{h k}{\epsilon_{n-\mu}}=a_{\mu}^{h k}, \\
& \mu>\frac{1}{2} n \text {; } \\
& \epsilon_{n-\mu}^{h k}=(-1)^{\mu(n+1)} a_{\mu}^{h k}, \quad \mu \leqq \frac{1}{2} n .
\end{aligned}
$$

(b) $\mu=\frac{1}{2} n=$ an odd integer.

Then

$$
\epsilon_{n / 2}^{2 h-1, k}=-a_{n / 2}^{2 h, k}, \quad \epsilon_{n / 2}^{2 h, k}=a_{n / 2}^{2 h-1, k} .
$$

We obtain for $\Gamma_{n}$ the following relations :

(a) $n \neq 4, \bmod 2$.

$$
\begin{aligned}
\Gamma_{n} \approx & \sum_{0 \leqq \mu<n / 2} \sum_{i, j=1}^{R_{\mu}} a_{n-\mu}^{i j} \cdot \gamma_{\mu}^{i} \times \delta_{n-\mu}^{j} \\
& +\sum_{\mu \leq n / 2}(-1)^{(n+1) \mu} \sum_{i, j=1}^{R_{\mu}} a_{n-\mu}^{i j} \cdot \gamma_{\mu}^{i} \times \delta_{n-\mu}^{i} .
\end{aligned}
$$

(b) $n \equiv 4, \bmod 2$.

$$
\begin{aligned}
\Gamma_{n} \approx & \sum_{0 \leqq \mu<n / 2} \sum_{i, j=1}^{R_{\mu}}\left(a_{n-\mu}^{i j} \cdot \gamma_{\mu}^{i} \times \delta_{n-\mu}^{j}+(-1)^{\mu} a_{\mu}^{i j} \cdot \gamma_{n-\mu}^{i} \times \delta_{\mu}^{j}\right) \\
& +\sum_{h=1}^{i R_{n} / 2} \sum_{k=1}^{R_{n} / 2}\left(a_{n / 2}^{2 h-1} \cdot \gamma_{n / 2}^{2 h} \times \delta_{n / 2}^{k}-a_{n / 2}^{2 h, k} \cdot \gamma_{n / 2}^{2 h-1} \times \delta_{n / 2}^{k}\right) .
\end{aligned}
$$


65. A new proof of Veblen's theorem on fundamental sets. We refer to the theorem of Part I, $\S 6$, according to which the invariant factors of the matrix

$$
\left\|\left(\gamma_{\mu}^{i} \cdot \gamma_{n-\mu}^{i}\right)\right\|
$$

for any two fundamental sets are all unity. Let them be in any case $e_{1}$, $e_{2}, \cdots, e_{R_{\mu}}$. That their number is $R_{\mu}$ follows from other considerations that need not detain us. ${ }^{*}$ The reduction to canonical sets will be accomplished as before, only now

$$
\left(\gamma_{\mu}^{i} \cdot \gamma_{n-\mu}^{i}\right)= \pm e_{i},
$$

the same indices being zero as previously. Therefore we have now

$$
\stackrel{h k}{\epsilon_{n-\mu}} \cdot e_{h} e_{k}= \pm a_{\mu}^{h k} \cdot e_{k} .
$$

Hence $e_{h}$ is a factor of $a_{\mu}^{h k}$, and in particular of $a_{\mu}^{h h}$ for every $T$. But for the identical transformation $a_{\mu}^{h h}=1$, hence $e_{h}=1$ which is precisely Veblen's theorem.

\section{§3. COINCIDENCES AND FIXED POINTS OF TRANSFORMATIONS OF A MANIFOLD}

66. A coincidence of two transformations $T, T^{\prime}$ is a pair of points $A, A^{\prime}$, of $M_{n}$, such that $A^{\prime}$ is a transform of $A$, by both $T$ and $T^{\prime}$. A fixed point of $T$ is a coincidence for $T$ and the identical transformation. Let $\Gamma_{n}, \Gamma_{n}{ }^{\prime}$ be the cycles corresponding to $T$ and $T^{\prime}$, and let $B$ be the image of $A^{\prime}$ on $M_{n}{ }^{\prime} ; A \times B$ is an intersection of $\Gamma_{n}$ with $\Gamma_{n}{ }^{\prime}$ and conversely to such an intersection corresponds a coincidence of $T$ and $T^{\prime}$. The determination of the number of coincidences and fixed points is then reduced to a question of intersections of cycles. The actual numbers are not definite, may even vary for transformations of the same class, become infinite, and so on. Not so, however, with the attached Kronecker index $\left(\Gamma_{n} \cdot \Gamma_{n}{ }^{\prime}\right)$, whose determination alone is usually possible. Its interpretation is simple enough. If we consider suitable approximations to $T$ and $T^{\prime}$ there will be only a finite number of intersections. Some of these, say $k^{\prime}$, shall be counted positively, others, say $k^{\prime \prime}$, counted negatively according to a definite rule, and the difference $k^{\prime}-k^{\prime \prime}$ is independent of the mode of approximation, in a sense sufficiently clear in the light of Part I, §5. From what has been established there, it follows that the number of coincidences to be obtained in the sequence is an actual topological invariant of the transformations involved, the same being

\footnotetext{
* See my Monograph, p. 13; also Veblen's paper on this question quoted in Part I, \$6.
} 
true for the number of fixed points counted in an analogous manner to the coincidences.

67. We shall now endeavor to characterize topologically the coincidences according to the signs of their contributions to $\left(\Gamma_{n} \cdot \Gamma_{n}{ }^{\prime}\right)$. We select two polyhedral approximations that intersect in a finite number of points only, in such a way that if $A \times B$ is one of them it has neighborhoods on the cycles and on $M_{n} \times M_{n}^{\prime}$ that are interiors of simplexes. We continue to call the approximations $\Gamma_{n}, \Gamma_{n}^{\prime}$. Each intersection $A \times B$ counts for \pm 1 in computing the index, and as far as its neighborhood alone and those of $A$ and $B$ on $M_{n}$ and $M_{n}{ }^{\prime}$ are concerned, everything is as if all complexes involved were linear spaces. The matrix-indicatrix method will then be again the most convenient.

To $\Gamma_{n}$ and $\Gamma_{n}{ }^{\prime}$ we may make correspond matrices

$$
\left\|X_{n}, Y_{n}\right\|,\left\|X_{n}^{\prime}, Y_{n}^{\prime}\right\| \text {. }
$$

Regarding $X_{n}$ and $X_{n}^{\prime}$ we may replace them by any others whose determinants have the same signs. This is an immediate corollary of the definition of the indicatrix. Let us assume our coördinate system such that the unit matrix of order $n, I_{n}$, corresponds to an indicatrix for $M_{n}$ as referred to the $x$ coördinates. Then $I_{n}$ will play the same part for $M_{n}{ }^{\prime}$ and the $y$ coördinates, $I_{2 n}$ for $M_{n} \times M_{n}^{\prime}$ and the coördinates $x, y$. Under the circumstances, as we have shown in No. 60, if $\theta, \theta^{\prime}$ denote the contributions of $A \times B$ to the indices $\left(\Gamma_{n} \cdot A \times M_{n}{ }^{\prime}\right),\left(\Gamma_{n}^{\prime} \cdot A \times M_{n}^{\prime}\right)$, then the determinants in question have the sign of $\theta$ and $\theta^{\prime}$. We conclude that there will be suitable indicatrices for $\theta \Gamma_{n}$ and $\theta^{\prime} \Gamma_{n}^{\prime}$ of type

$$
\left\|I_{n}, Y_{n}\right\|,\left\|I_{n}, Y_{n}^{\prime}\right\| \text {. }
$$

Now the contribution of $A \times B$ to $\left(\theta \Gamma_{n} \cdot \theta^{\prime} \Gamma_{n}{ }^{\prime}\right)$, in absolute value equal to unity, has the sign of

$$
\left|\begin{array}{ccc}
I_{n} & , & Y_{n} \\
I_{n} & , & Y_{n}^{\prime}
\end{array}\right|,
$$

since $I_{2 n}$ is a matrix-indicatrix for $M_{n} \times M_{n}{ }^{\prime}$. This determinant is equal to $\left|Y_{n}{ }^{\prime}-Y_{n}\right|$, and therefore the contribution of $A \times B$ to $\left(\Gamma_{n} \cdot \Gamma_{n}{ }^{\prime}\right)$ has the sign of $\theta \theta^{\prime}\left|Y_{n}^{\prime}-Y_{n}\right|$. The determinant factor is certainly $\neq 0$, else $A \times B$ would not be an isolated intersection of the $\Gamma^{\prime}$ 's. Furthermore owing to the degree of arbitrariness in the choice of these cycles we may always assume that the equation of the $n$th degree

$$
f(t)=\left|Y_{n}^{\prime}-t Y_{n}\right|=0
$$


(characteristic equation of $Y_{n}{ }^{\prime}-t Y_{n}$ ) has only distinct roots. We know already that $f(1) \neq 0$.

Let $e= \pm 1$ be the same integer as in No. $60(+1$ if $T$ maintains an indicatrix of $M_{n}$ at $A,-1$ if it inverts it). Then as shown there

$$
\epsilon\left|Y_{n}\right|>0 \text {. }
$$

Since the determinant $Y_{n}$ is the leading coefficient of $f(t), f(1)$ has the sign of $(-1)^{\nu} \epsilon$, where $\nu$ is the number of real roots of $f(t)$ that are less than unity. Therefore the contribution of $A \times B$ to $\left(\Gamma_{n} \cdot \Gamma_{n}{ }^{\prime}\right)$ is equal to $(-1)^{\nu} \epsilon \theta \theta^{\prime}$.

68. It is decidedly desirable and worth while to find a geometric interpretation for $\nu$. The transformation $T$ acts as an affine transformation whereby the vector $V$ whose $x$ components are $\xi_{1}, \xi_{2}, \cdots, \xi_{n}$ is changed into a vector whose components are defined by the matrix product.

$$
\left\|\xi_{1}, \xi_{2}, \cdots, \xi_{n}\right\| \cdot Y_{n},
$$

and similarly for $T^{\prime}$ and $Y_{n}^{\prime}$. Let $t_{i}$ be a real root of $f(t)$. Then there is a $V_{i}$ whose transforms by $T$ and $T^{\prime}$ are collinear and in the ratio $1: t_{i}$. Let $V_{1}, V_{2}, \cdots, V_{\nu}$ be the similar vectors for all real roots $<1$. The vectors

$$
u_{1} V_{2}+\cdots+u_{\nu} V_{\nu} ; \quad 0 \leqq u_{i} \leqq 1, \sum u_{i}=1
$$

fill up a simplex $\Sigma_{\nu}^{0}$ of $M_{n}$ with a vertex at $A$. Its transforms by $T$ and $T^{\prime}$ are simplexes $\Sigma_{v}, \Sigma_{\nu}^{\prime}$ with the common vertex $A^{\prime}$, the second being entirely on the first, with all cells through $A^{\prime}$ situated in the same linear spaces, and $\nu$ is the largest integer for which such simplexes exist. This is the desired interpretation for $\nu$.

69. We now proceed with the determination of $\left(\Gamma_{n} \cdot \Gamma_{n}{ }^{\prime}\right)$.

Referring to No. 54, and recalling the special relations for canonical sets, we find that the only indices $\left(\gamma_{\lambda}^{i} \times \bar{\delta}_{n-\lambda}^{j} \cdot \gamma_{\mu}^{h} \times \delta_{n-\mu}^{k}\right) \neq 0$ are those for which $\lambda+\mu=n$ and whose values are given below.

(a) $\mu \neq \frac{1}{2} n$ or else $=\frac{1}{2} n$ an even integer.

$$
\begin{aligned}
\left(\gamma_{\mu}^{i} \times \delta_{n-\mu}^{j} \cdot \gamma_{n-\mu}^{i} \times \delta_{\mu}^{j}\right) & =(-1)^{n(\mu+1)}\left(\gamma_{\mu}^{i} \cdot \gamma_{n-\mu}^{i}\right)\left(\gamma_{\mu}^{j} \cdot \gamma_{n-\mu}^{j}\right) \\
& =(-1)^{n(\mu+1)} ; 1 \leqq i, j \leqq R_{\mu},
\end{aligned}
$$

for with canonical systems, the two indices dropped are equal, their common value being \pm 1 .

(b) $\mu=\frac{1}{2} n$ an odd integer. For entirely similar reasons the indices below alone are left, with values as given:

$$
\begin{aligned}
& \left(\gamma^{2 h-1} \times \delta^{2 k-1} \cdot \gamma^{2 h} \times \delta^{2 k}\right)=\left(\gamma^{2 h} \times \delta^{2 k} \cdot \gamma^{2 h-1} \times \delta^{2 k-1}\right)=-1, \\
& \left(\gamma^{2 h-1} \times \delta^{2 k} \cdot \gamma^{2 h} \times \delta^{2 k-1}\right)=\left(\gamma^{2 h} \times \delta^{2 k-1} \cdot \gamma^{2 h-1} \times \delta^{2 k}\right)=+1 .
\end{aligned}
$$


To facilitate the reading of these formulas we have omitted the lower indices whose common value is $\frac{1}{2} n$.

70. The application to $\left(\Gamma_{n} \cdot \Gamma_{n}{ }^{\prime}\right)$ is immediate. Let, throughout, the $\eta$ 's and $\beta$ 's play the same part for $\Gamma_{n}^{\prime}$ or $T^{\prime}$ as the $\epsilon$ 's and $a^{\prime}$ 's for $\Gamma_{n}$ or $T$. The distributive law for indices gives

$$
\left(\Gamma_{n} \cdot \Gamma_{n}^{\prime}\right)=\sum_{i, j, \mu} \epsilon_{n-\mu}^{i j} \eta_{\mu}^{n k}\left(\gamma_{\mu}^{j} \sim \delta_{n-\mu}^{j} \cdot \gamma_{n-\mu}^{k} \times \delta_{\mu}^{k}\right)
$$

Therefore for $n \neq 2, \bmod 4$, and with $\mu$ replaced by $n-\mu$,

$$
\left(\Gamma_{n} \cdot \Gamma_{n}^{\prime}\right)=\sum_{\mu=0}^{n}(-1)^{n \mu} \sum_{i, j=1}^{R_{\mu}} \epsilon_{n-\mu}^{i j} \eta_{\mu}^{i j},
$$

while for $n \equiv 2, \bmod 4$, after some simplifications,

(70.2) $\left(\Gamma_{n} \cdot \Gamma_{n}{ }^{\prime}\right)=$ (same sum as in (70.1) except that $\mu$ does not take the value $\left.\frac{1}{2} n\right)$

$$
\begin{aligned}
& +\sum_{n, k=1}^{1 R_{n / 2}}\left(\epsilon_{n / 2}^{2 h-1,2 k} \eta_{n / 2}^{2 h, 2 k-1}\right. \\
& \left.+\epsilon_{n / 2}^{2 h, 2 k-1} \eta_{n / 2}^{2 h-1,2 k}-\epsilon_{n / 2}^{2 h-1,2 k-1} \eta_{n / 2}^{2 h, 2 k}-\epsilon_{n / 2}^{2 h, 2 k} \eta_{n / 2}^{2 h-1,2 h-1}\right) .
\end{aligned}
$$

In terms of the $a$ 's and $\beta$ 's, by means of No. 64 we find, if $n \neq 2, \bmod 4$,

$$
\left(\Gamma_{n} \cdot \Gamma_{n}^{\prime}\right)=\sum_{\mu=0}^{n}(-1)^{\mu} \sum_{i, j=1}^{R \mu} a_{\mu}^{i j} \beta_{n-\mu}^{i j},
$$

and if $n \equiv 2, \bmod 4$,

(70.4) $\left(\Gamma_{n} \cdot \Gamma_{n}{ }^{\prime}\right)=$ (same sum as in (70.3) except that $\mu$ does not take the value $\left.\frac{1}{2} n\right)$

$$
\begin{aligned}
& +\sum_{n, k=1}^{1} R_{n / 2}^{2 h-1,2 k} \beta_{n / 2}^{2 h, 2 k-1} \\
& \left.+a_{n / 2}^{2 h, 2 k-1} \beta_{n / 2}^{2 h-1,2 k}-a_{n / 2}^{2 h-1,2 k-1} \beta_{n / 2}^{2 h, 2 k}-a_{n / 2}^{2 h, 2 k} \beta_{n / 2}^{2 h-1,2 k-1}\right) .
\end{aligned}
$$

71. To obtain the number of invariant points of $T$, always counted with a certain sign, it is convenient to replace throughout $T$ by the identical transformation, whose cycle we denote by $\Gamma_{n}^{0}$, and $T^{\prime}$ by $T$. The homologies for the identical transformation are (No. 62)

$$
\bar{\gamma}_{\mu}^{h} \sim \gamma_{\mu}^{h},
$$


hence $a_{\mu}^{\prime \prime}=1, a_{\mu}^{i j}=0$ if $i \neq j$. On replacing the $a$ 's by these values and the $\beta$ 's by the $a$ 's both (70.3) and (70.4) reduce to the very simple formula

$$
\left(\Gamma_{n}^{0} \cdot \Gamma_{n}\right)=\sum_{\mu=0}^{n}(-1)^{\mu} \sum_{i=1}^{R_{\mu}} a_{\mu}^{i j}
$$

Remarkably enough this formula is still correct if the fundamental sets cease to be canonical. For if $\gamma_{\mu}^{\prime \prime}$ is a new fundamental set, we have

$$
\gamma_{\mu}{ }^{i} \approx \sum_{j} A_{i j} \gamma_{\mu}^{j}
$$

$\left(\bmod M_{n}\right)$

where the $A$ 's are integers whose determinant

$$
\left|A_{i j}\right|= \pm 1 \text {. }
$$

Hence the transformation matrix of the $\gamma^{\prime}$ 's that takes the place of

$$
\left\|a_{\mu}^{i j}\right\|
$$

is obtained by transforming the latter by means of the matrix of the $A$ 's, an operation which leaves unchanged the sum of the terms in the principal diagonal, which is the term corresponding to $\mu$ in (71.1).

A particularly noteworthy case is when the effect of $T$ on any cycle is merely to increase it by a zero-divisor, which includes as a special case deformations. Then all the $a$ 's in (71.1) are equal to +1 and

$$
\left(\Gamma_{n} \cdot \Gamma_{n}\right)=\sum_{\mu=0}^{n}(-1)^{\mu} R_{\mu}
$$

This expression is the well known Euler-Poincare characteristic number of $M_{n}$ (difference between the number of cells of even dimensionality of any covering $C_{n}$ and the number of the rest). Since $R_{\mu}=R_{n-\mu}$ its value is zero for $n$ odd, hence this very neat proposition : for a $T$ of the preceding type, in particular of the same class as the identity, the number of invariant points counted with their signs is equal to the Euler-Poincaré characteristic. It is zero when $n$ is odd.

Thus for $n=2$ the number is $2-2 p$ as found by Birkhoff, who however confined himself to analytic transformations.

Fixed points for transformations of hyperspheres have proved of importance in many questions. There are then no cycles of dimensions other than 0 or $n$ so that (71.1) becomes

$$
\left(\Gamma_{n}^{0} \cdot \Gamma_{n}\right)=a_{0}+(-1)^{n} a_{n},
$$

a result obtained by Brouwer for $a_{0}=1$. 
Finally formula (71.1) for $n=2$ includes practically all related results obtained in recent years by various authors (Brouwer, Nielsen, Kérékjárto, Alexander).

72. Another class of applications is to coincidences of algebraic correspondences on algebraic curves. Owing to a proposition which I proved in my Monograph, p. 19, the Kronecker indices give then the exact number of intersections. We should therefore expect to be able to identify (70.4) with the well known coincidence formula due to A. Hurwitz.* Observe that his $h_{k i}^{\prime}$, etc., are the same as the $h_{k i}$, etc., not for $C^{\prime}$ but for $C^{\prime-1}$ and should be replaced as indicated in his footnote; for example, $h_{\mathbf{a}}^{\prime}$ by $G_{\mathbf{k}}^{\prime}$ and so on. With these changes and a comparison of notations the identification becomes complete.

Thus the coincidence formulas may be obtained without making use of any function theory. The classical Hurwitz relations between the periods are simply the analytical translation of the transformations on the linear cycles of $M_{n}$.

* Mathematische Annalen, vol. 28 (1887), p. 578, formula 35. See in this connection Chisini, Istituto Lombardo Rendiconti, ser. 2, vol. 7 (1924), p. 481.

UNIVERSITY OF KANSAS,

LAWRENCE, KANSAS 